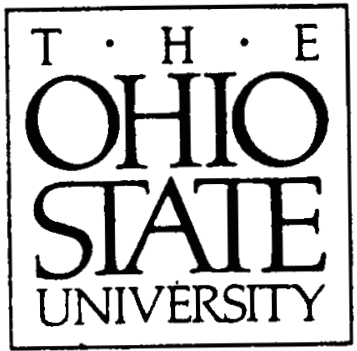

428

\title{
A Method to Design Blended Rolled Edges for Compact Range Reflectors
}

\author{
Inder J. Gupta and Walter D. Burnside
}

The Ohio State University

ElectroScience Laboratory

Department of Eloctrical Engineering

Columbus, Ohio 43212

Technical Report 719493-6

Grant No. NSG 1613

March 1989

National Aeronautics and Space Administration

Langley Research Center

Hampton, VA 22217

(NASI-CR-184976) A AEIHOD TC CESIGE BLEEDED

FCLLED EDGES FOE CCEFICT EAME EEFLECTCRS

$N 89-23736$

(Chio state Driv.): $42 \mathrm{~F}$

CSCL 13H 


\section{NOTICES}

When Government drawings, specifications, or other data are used for any purpose other than in connection with a definitely related Government procurement operation, the United States Government thereby incurs no responsibility nor any obligation whatsoever, and the fact that the Government may have formulated, furnished, or in any way supplied the said drawings, specifications, or other data, is not to be regarded by implication or otherwise as in any manner licensing the holder or any other person or corporation, or conveying any rights or permission to manufacture, use, or sell any patented invention that may in any way be related thereto. 
80272-101

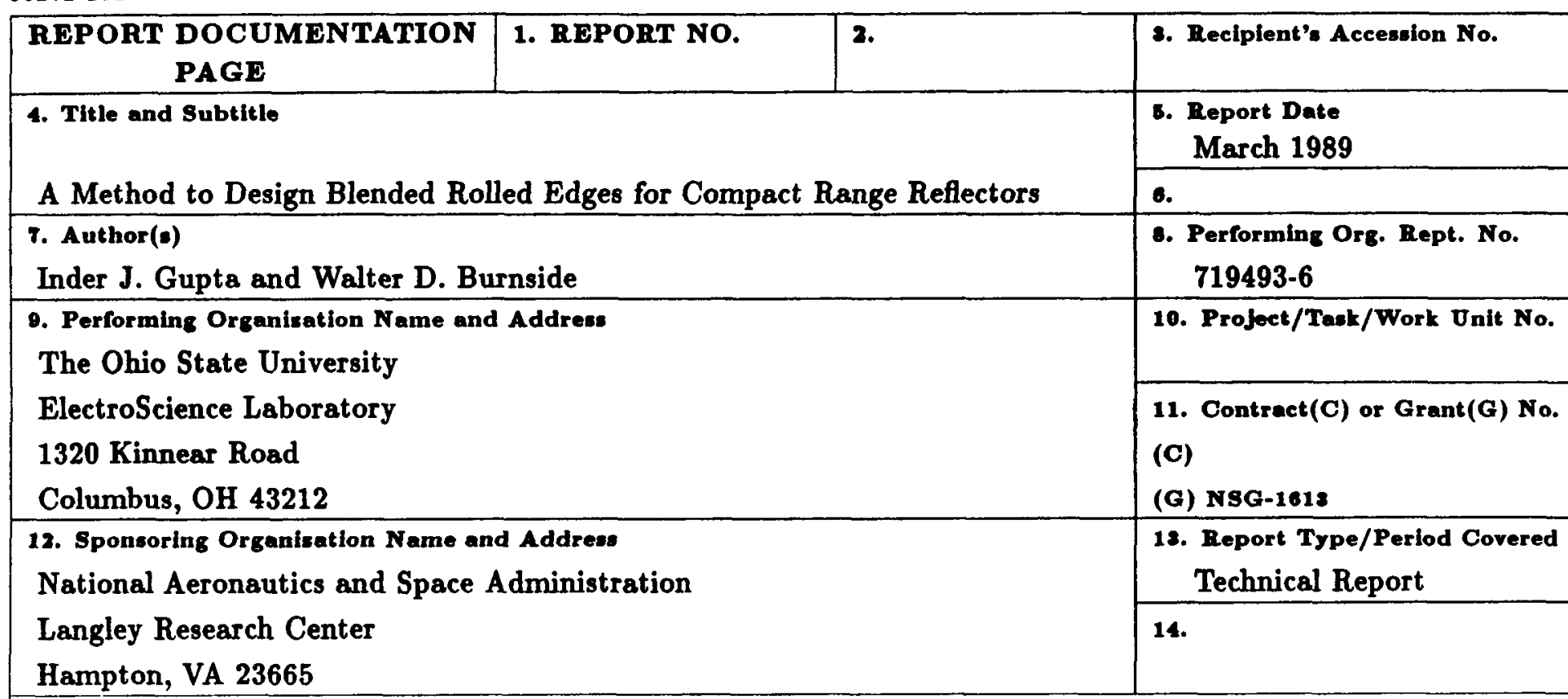

16. Supplementary Notes

16. Abstract (Limit, 200 words)

A method to design blended rolled edges for arbitrary rim shape compact range reflectors is presented. The reflectors may be center-fed or offset-fed. The method leads to rolled edges with minimal surface discontinuities. It is shown that the reflectors designed using the prescribed method can be defined analytically using simple expressions. A procedure to obtain optimum rolled edges parameter is also presented. The procedure leads to blended rolled edges that minimize the diffracted fields emanating from the junction between the paraboloid and the rolled edge surface while satisfying certain constraints regarding the reflector size and the minimum operating frequency of the system.

17. Document Analysis a. Descriptors

b. Identifiers/Open-Ended Terms

c. Cosati Field/Group

18. A vailability Statement

10. Security Class (Thin Report) Unclassified

20. Security Class (This Paze)

Unclassified 
Contents

LIST OF FIGURES

iv

SECTION

PAGE

1 INTRODUCTION 1

2 BLENDED ROLLED EDGE CONCEPT 4

3 METHOD TO SELECT THE ROLLED EDGE PARAMETERS

4 BLENDED ROLLED EDGES FOR 3-D REFLECTORS 18

5 DESIGN EXAMPLE 25

6 SUMMARY AND CONCLUSION 32 


\section{List of Figures}

1 Parabola and rolled edge coordinate system. The junction is located at $\rho=\rho_{j} . \ldots \ldots \ldots \ldots \ldots \ldots \ldots$

2 Elliptical rolled edge parameters. . . . . . . . . . 6

3 Blended rolled edge parameters. . . . . . . . . . . 8

4 Error $\left(\epsilon_{n}^{2}\right)$ versus $a_{e}$ when $\gamma_{m}$ is fixed and $b_{e}$ and $x_{m}$ are chosen to satisfy the two constraints. . . . . . . . . . 13

5 Error $\left(\epsilon_{n}^{2}\right)$ versus $a_{e}$ for a 24 foot focal length reflector. $\rho_{j}=$ $15^{\prime}$, rolled edge height $=5^{\prime}$, minimum frequency $=1 \mathrm{GHz}$, cosine blending. . . . . . . . . . . . . . . 14

6 Junction diffracted fields for a 24 foot focal length reflector for various rolled edges. Frequency $=1 \mathrm{GHz}, z$-displacements $=50^{\prime}$ and a magnetic line source feed. . . . . . . . . . 16

7 Paraboloid coordinate system and the junction contour for a compact range reflector. . . . . . . . . . . 19

8 Various coordinate systems. $\left(x_{a v g}, y_{\text {avg }}, z_{\text {avg }}\right)$ is the center of the compact range reflector. . . . . . . . . . . 21

9 The section of the paraboloid in the rolled edge plane $\left(\rho^{\prime} z^{\prime}\right)$. Vertex of the equivalent parabola coincides with the origin of $\left(\rho^{\prime \prime} z^{\prime \prime}\right)$ coordinate system. . . . . . . . . . . .

10 Front view of the junction contour of a concave edge compact range reflector with $x_{\text {left }}=-4^{\prime}, x_{\text {right }}=4^{\prime}, y_{\text {bottom }}=5.5^{\prime}$, and $y_{\text {top }}=11.5^{\prime}$. The focal length is $24^{\prime}$ and $r_{e}=3.5^{\prime} . \ldots 26$

11 Junction height versus $\phi^{\prime}$ for the concave edge reflector. . . 27

12 Front view of the concave edge reflector with cosine squared blended rolled edges. Rolled edge height $=3.5^{\prime}$, minimum frequency $=2 \mathrm{GHz} . \ldots \ldots \ldots \ldots \ldots$

13 Scattered fields of the concave edge reflector in the target zone along two vertical cuts. $z$-displacement $=20^{\prime}$, Frequency $=2 \mathrm{GHz}$, Subreflector feed. . . . . . . . . .

14 Scattered fields of the concave edge reflector along three horizontal cuts. $z$-displacement $=20^{\prime}$, Frequency $=2 \mathrm{GHz}$, Subreflector feed. . . . . . . . . . . . . . . 31

15 Target zone, defining rectangle and the junction contour. . 34 


\section{INTRODUCTION}

For accurate radar cross section (RCS) or antenna pattern measurements, one should illuminate the scattering body or antenna under test with a plane wave. A compact range is an electromagnetic system used to simulate the plane wave. In a compact range, a paraboloid is normally used as the main reflector which converts the spherical wavefront of a point source located at the focal point of the paraboloid into a planar wavefront. However, since a finite size reflector is used in a compact range, there are diffracted fields which emanate from the rim of the reflector. These diffracted fields distort the planar wavefront and lead to erroneous measurements. In order to reduce the diffracted fields, various edge terminations have been investigated such as absorber material [1], serrated edges [2,3], shaped reflectors [4] and rolled edges [5]. Among these approaches, rolled edge terminations can provide the lowest diffracted fields for a given size reflector. Using the rolled edge concept, an elliptical or some similar convex surface is added to the paraboloid along its rim such that the surface is smooth and continuous. The addition of the rolled edge reduces the magnitude of the discontinuity in the specular reflected field as the specular point moves from the parabola onto the rolled edge. This in turn reduces the edge diffracted fields coming from the termination of the parabola. Also, since the specular reflection from the rolled edge is directed away from the potential target zone, it does not distort the planar wave front.

The original elliptical rolled edge [5] had a large discontinuity in the 
reflected fields across the junction between the parabola and the rolled edge. This discontinuity resulted from the fact that the radius of curvature of a compact range reflector with a simple rolled edge is discontinuous at the junction between the paraboloid and the rolled edge. To decrease the discontinuity in the radius of curvature, one can increase the semi-major axis of the ellipse $\left(a_{e}\right)$ and/or decrease the semi-major axis of the ellipse $\left(b_{e}\right)$. An increase in $a_{e}$, will, however, make the rolled edge too large; while, a decrease in $b_{e}$ will make the rolled edge termination look like a knife edge, especially at low frequencies, which is undesirable. Thus, $a_{e}$ and $b_{e}$ should be chosen such that the total height of the reflector is within a specified limit and the minimum radius of curvature of the rolled edge is at least one fourth of a wavelength at the lowest frequency of operation. This choice of $a_{e}$ and $b_{e}$ will satisfy the design constraints but may lead to diffracted fields (from junction between the rolled edge and the paraboloid) which are too large for certain applications.

Recently, Burnside et al. [6] introduced the concept of blended rolled edges, which further reduces the diffracted fields. In a blended rolled edge, a part of the elliptical rolled edge is blended with an extension of the parabola to form the rolled edge. The blending is done such that the rolled edge looks like the parabola near the junction and like the ellipse at the other end. Pistorius [7] showed that with a blended rolled edge one can make the radius of curvature and a certain number of its derivatives continuous across the junction, which in turn leads to very small diffracted fields. Again, one should choose the blended rolled edge parameters such that the 
design constraints of the maximum total height and the minimum radius of curvature are met. It can be shown that in the case of a blended rolled edge there is infinite sets of parameters which satisfy these design constraints. One would normally like to choose the values which minimize the diffracted fields. In this paper, a method to find the optimum rolled edge parameters is given. The method is applicable to arbitrary rim shape reflectors which may be center-fed (the center of the reflector is on the axis of the paraboloid) or offset-fed. The design procedure leads to a reflector which is smooth and continuous and satisfies the constraints regarding the reflector size and the minimum radius of curvature. Using the design procedure, blended rolled edge for an offset-fed concave edge [8] reflector is designed. The performance of the reflector in terms of the scattered fields in the target zone is also presented.

The rest of the paper is organized as follows. In section II, the concept of blended rolled edges as applied to two dimensional reflectors is presented. In section III, a method to select rolled edge parameters for two dimensional systems is discussed. In section IV, the rolled edge plane for three-dimensional reflectors in defined and analytic expressions for the whole reflector surface (including blended rolled edges) are given. Section V contains a design example. Finally, section VI contains a summary and general conclusions. 


\section{ORIGINAL PAGE IS}

OF POOR QUALTY

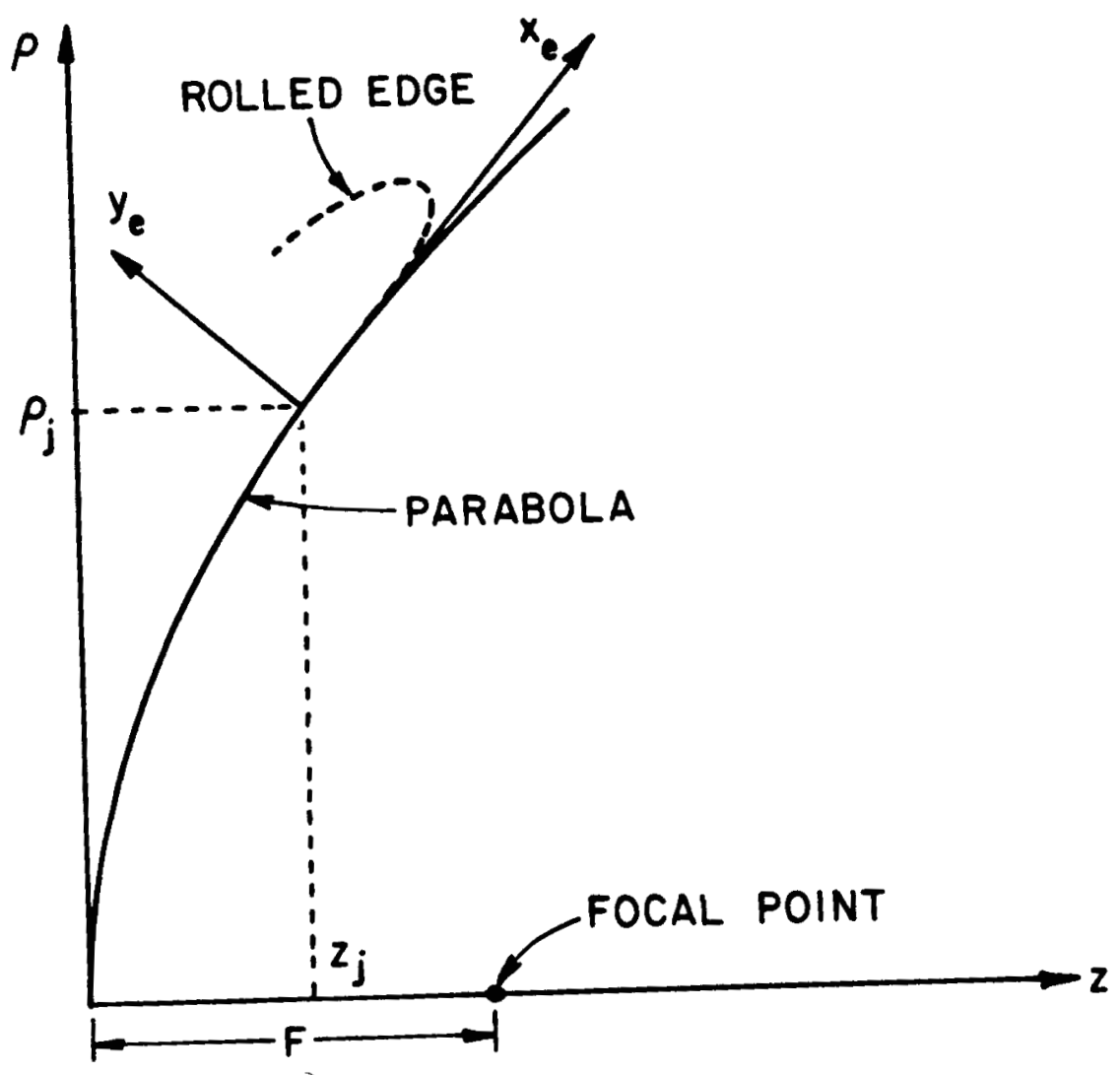

Figure 1: Parabola and rolled edge coordinate system. The junction is located at $\rho=\rho_{j}$.

\section{BLENDED ROLLED EDGE CONCEPT}

Let a parabola of focal length $F$ be defined in the $(\rho z)$ plane as shown in Figure 1. Then the defining equation for the parabola is given by

$$
z=\rho^{2} / 4 F
$$

If one wants to add an elliptical rolled edge to this parabola in the positive $\rho$ direction at a point $\rho=\rho_{j}$ such tha $\cdots$ esulting total surface is smooth and continuous, then the rolled eds ordinate system $\left(x_{e}, y_{e}\right)$ should be 
defined as (see Figure 1)

$$
\begin{gathered}
\hat{x}_{e}=x_{p 2} \hat{\rho}+x_{p 3} \hat{z}, \text { and } \\
\hat{y}_{e}=y_{p 2} \hat{\rho}+y_{p 3} \hat{z}
\end{gathered}
$$

where

$$
\begin{gathered}
x_{p 2}=2 F /\left(\rho_{j}^{2}+4 F^{2}\right)^{1 / 2} \\
x_{p 3}=\rho_{j} /\left(\rho_{j}^{2}+4 F^{2}\right)^{1 / 2} \\
y_{p 2}=\rho_{j} /\left(\rho_{j}^{2}+4 F^{2}\right)^{1 / 2}, \text { and } \\
y_{p 3}=-2 F /\left(\rho_{j}^{2}+4 F^{2}\right)^{1 / 2} .
\end{gathered}
$$

Note the $\hat{x}_{e}$ is the tangent to the parabola at the junction point, and $\hat{y}_{e}$ is the outward normal to the parabola at the junction point. Using Equations (4) - (7), the coordinate transformation between the $\left(x_{e}, y_{e}\right)$ system and the $(\rho, z)$ system is given by

$$
\left(\begin{array}{c}
\rho \\
z
\end{array}\right)=\left(\begin{array}{ll}
x_{p 2} & y_{p 2} \\
x_{p 3} & y_{p 3}
\end{array}\right)\left(\begin{array}{c}
x_{e} \\
y_{e}
\end{array}\right)+\left(\begin{array}{c}
\rho_{j} \\
z_{j}
\end{array}\right)
$$

where $z_{j}=\rho_{j}^{2} / 4 F$. In the rolled edge coordinate system, the equation of the ellipse, as shown in Figure 2, is given by

$$
x_{e}(\gamma)=a_{e} \sin \gamma, \text { and }
$$




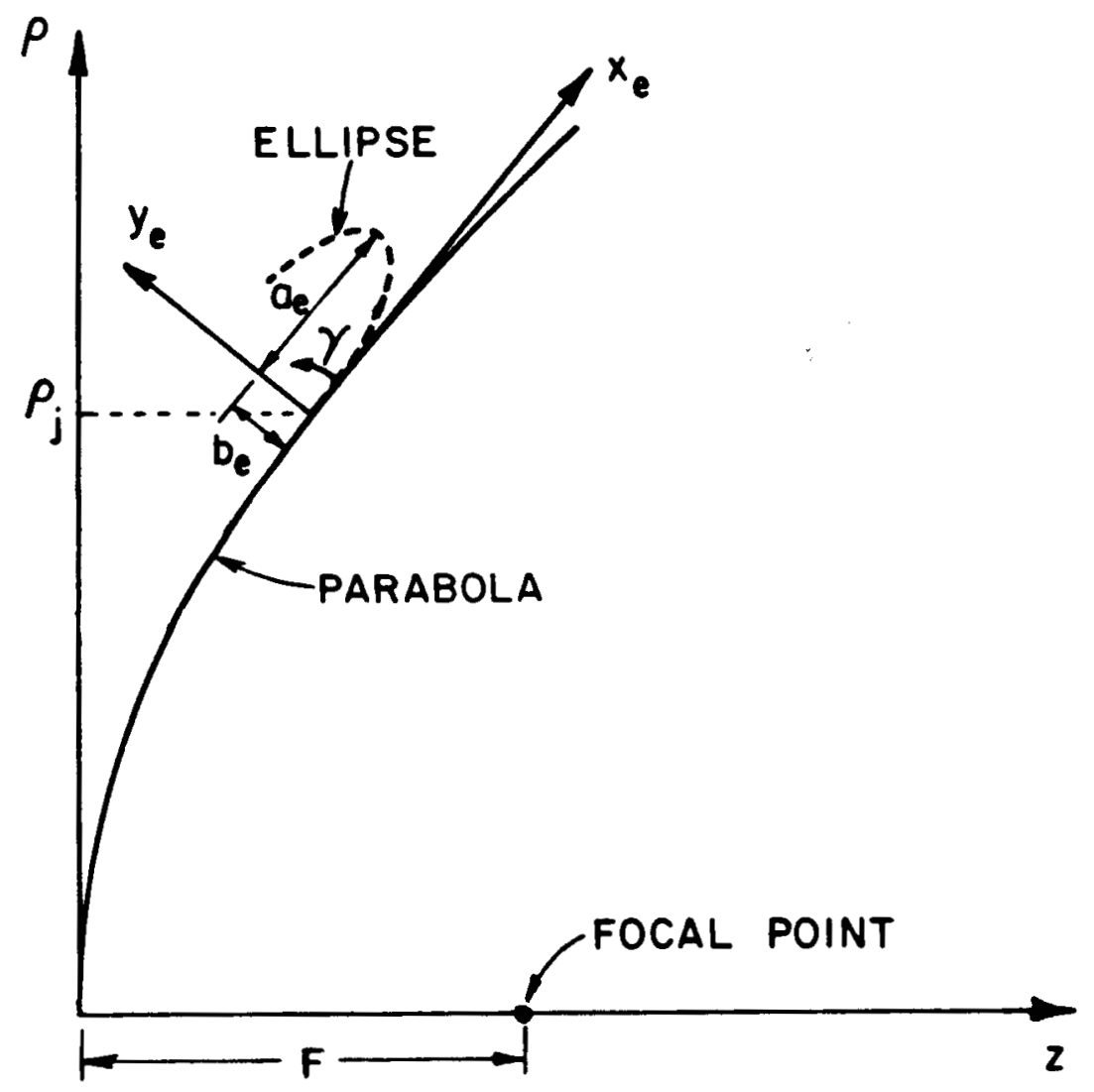

Figure 2: Elliptical rolled edge parameters. 


$$
y_{e}(\gamma)=b_{e}(1-\cos \gamma)
$$

where $a_{e}$ is the semi-major axis of the ellipse, $b_{e}$ is the semi-minor axis of the ellipse and $\gamma$ is a parametric angle such that $0 \leq \gamma \leq \gamma_{m}$. Note that $\gamma_{m}$ defines how much of the ellipse is used as the rolled edge. Normally $105^{\circ} \leq \gamma_{m} \leq 180^{\circ}$. It is obvious that the choice of $\gamma_{m}$ does not affect $a_{e}$ and $b_{e}$. In the $(\rho, z)$ coordinate system, the coordinates of the elliptical rolled edge are given by

$$
\begin{gathered}
\rho_{\text {ellipse }}(\gamma)=\left(a_{e} \sin \gamma\right) x_{p 2}+b_{e}(1-\cos \gamma) y_{p 2}+\rho_{j}, \text { and } \\
z_{\text {ellipse }}(\gamma)=\left(a_{e} \sin \gamma\right) x_{p 3}+b_{e}(1-\cos \gamma) y_{p 3}+z_{j}
\end{gathered}
$$

Note that the total surface is given in two parts. For $\rho \leq \rho_{j}$, the surface is a parabola as defined in Equation (1) while for $\rho>\rho_{j}$, the surface is given by Equations (11) and (12) as function of the parametric angle, $\gamma$. One can show the surface defined by (1) and (11) and (12) has a discontinuity in the radius of curvature at the junction point $\left(\rho_{j}, z_{j}\right)$. This discontinuity can lead to significant diffracted fields whose magnitude may be too large for certain applications. To reduce the diffracted field level, one may want to use a blended rolled edge. A blended rolled edge as shown in Figure 3 , is generated by blending the elliptical rolled edge with an extension of the paraboloid. The equation of the blended rolled edge $[7,8]$ in the $(\rho, z)$ coordinate system is then given by

$$
\rho_{b}(\gamma)=\rho_{\text {parabola }}(\gamma)[1-b(\gamma)]+\rho_{\text {ellipee }}(\gamma) b(\gamma) \text {, and }
$$




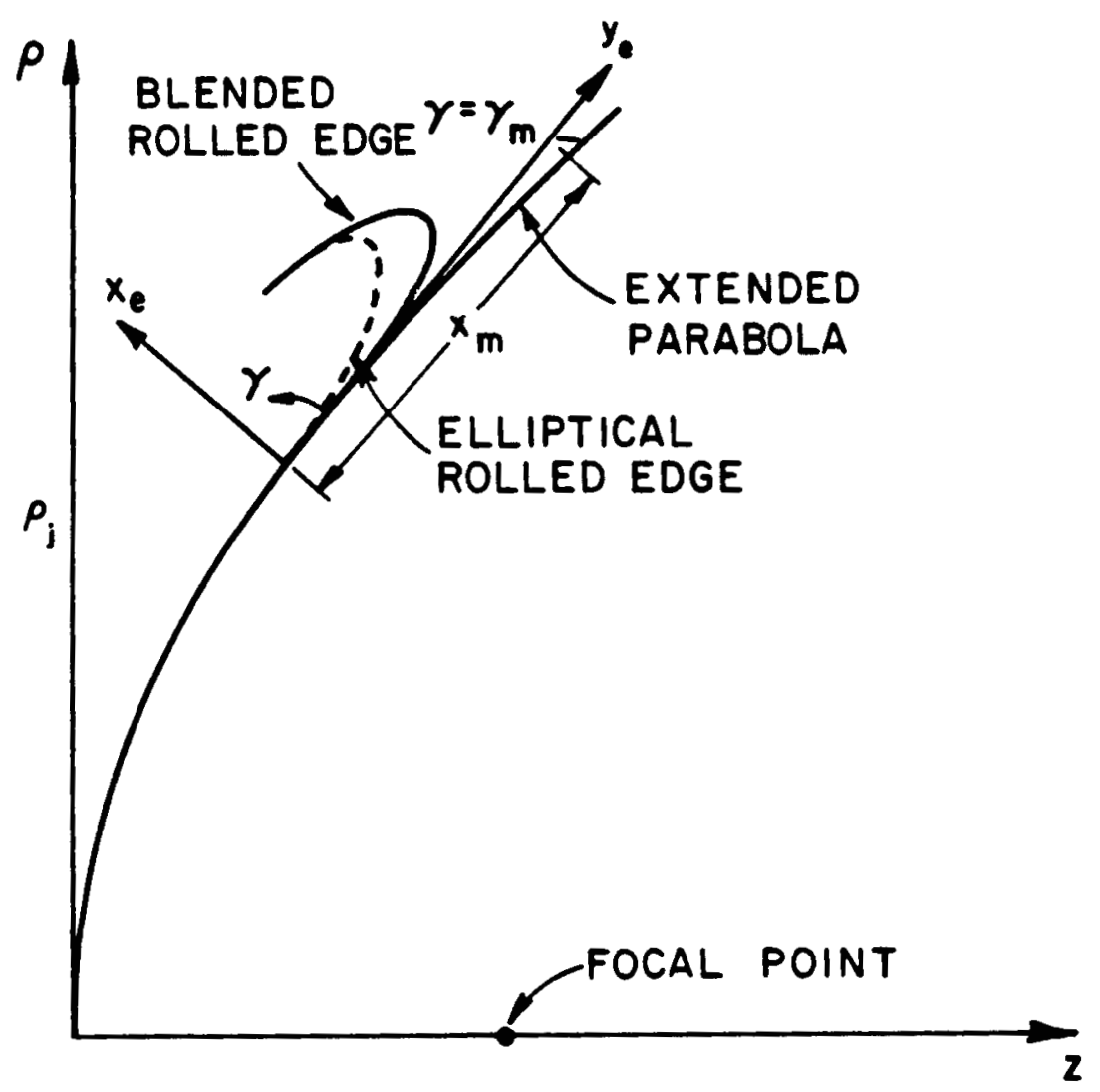

Figure 3: Blended rolled edge parameters. 


$$
z_{b}(\gamma)=z_{\text {parabola }}(\gamma)[1-b(\gamma)]+z_{\text {ellipse }}(\gamma) b(\gamma)
$$

where $\rho_{\text {parabola }}$ and $z_{\text {parabola }}$ are the coordinates of the extended parabola and $b(\gamma)$ is the blending function. The blending function varies between $[0,1]$ such that $b(0)=0$ and $b\left(\gamma_{m}\right)=1$. The extended parabola used in the blending is defined as

$$
\begin{gathered}
\rho_{\text {parabola }}(\gamma)=\gamma \frac{x_{m}}{\gamma_{m}} x_{p 2}+\rho_{j}, \text { and } \\
z_{\text {parabola }}(\gamma)=\rho_{\text {parabola }}^{2}(\gamma) / 4 F
\end{gathered}
$$

where $x_{m}$ defines the section of the parabola used in blending (see Figure 3). Substituting (11), (12), (15) and (16) into (13) and (14), one obtains

$$
\begin{array}{r}
\rho_{b}(\gamma)=\left(\gamma \frac{x_{m}}{\gamma_{m}} x_{p 2}\right)[1-b(\gamma)]+\left[\left(a_{e} \sin \gamma\right) x_{p 2}+\right. \\
\left.b_{e}(1-\cos \gamma) y_{p 2}\right] b(\gamma)+\rho_{j}
\end{array}
$$

and

$$
\begin{array}{r}
z_{b}(\gamma)=\left[\frac{\left(\gamma \frac{x_{m}}{\gamma_{m}} x_{p 2}\right)^{2}+2 \rho_{j} \gamma \frac{x_{m}}{\gamma_{m}} x_{p 2}}{4 F}\right][1-b(\gamma)]+ \\
{\left[\left(a_{e} \sin \gamma\right) x_{p 3}+b_{e}(1-\cos \gamma) y_{p 3}\right] b(\gamma)+z_{j}}
\end{array}
$$

Again the total surface is given in two parts. For $\rho \leq \rho_{j}$, the surface is a parabola as defined in Equation (1); while for $\rho \geq \rho_{j}$, the surface is given by Equations (17) and (18) as a function of the parametric angle, $\gamma$. Note that for a given junction height $\left(\rho_{j}\right)$ and focal length $(F)$, one can choose $a_{e}, b_{e}, x_{m}$ and $\gamma_{m}$ to satisfy various design constraints. In our application, 
there are two design constraints. One is that the total size (height) of the reflector should not exceed a specified limit, and the other is that the minimum radius of curvature of the rolled edge should not be less than $\lambda_{o} / 4$, where $\lambda_{o}$ is the wavelength at the lowest frequency of operation. It can be shown that there is a infinite set of values $a_{e}, b_{e}, x_{m}$ and $\gamma_{m}$ which will satisfy these two constraints. One wants to select the combination which will lead to the minimum diffraction from the junction between the blended rolled edge and the parabola. One way to do so is the cut and try approach which can be very time consuming and expensive. An efficient method of selecting the rolled edge parameters is given below.

\section{METHOD TO SELECT THE ROLLED EDGE PARAMETERS}

Let the blending function be chosen such that its first $n-1$ derivatives are zero at the junction and its $n^{\text {th }}$ derivative is non-zero; i.e.,

$$
\text { and } \left.\begin{array}{l}
b^{m}(0)=0, \quad m=0,1,2, \ldots n-1 \\
b^{n}(0) \neq 0
\end{array}\right\}
$$

Let us call such a function an $n^{\text {th }}$ order blending function. A list of such functions is given in [7]. For an $n^{\text {th }}$ order blending function, it can be shown [7] that the radius of curvature of the surface and its first $n-1$ derivatives

are continuous across the junction. The discontinuity in the $n^{\text {th }}$ derivative of the radius of curvature is given by

$$
\epsilon_{n}=\frac{\alpha F k^{n+3}}{\left(x_{m}\right)^{n}}\left[\frac{a_{e}}{\left(x_{m} / \gamma_{m}\right)}+\frac{F b_{e} k^{3}}{\left(x_{m} / \gamma_{m}\right)^{2}}-\frac{1}{2}\right]
$$


where

$$
k=\sqrt{1+\left(\rho_{j} / 2 F\right)^{2}}
$$

and $\alpha$ is a constant which depends on the type of blending function. Note that by selecting a proper combination of $a_{e}, b_{e}, x_{m}$ and $\gamma_{m}$, one can make $\epsilon_{n}$ also equal to zero. This will lead to a smoother surface which in turn should reduce the diffracted field magnitude. Thus, one should choose the rolled edge parameters such that $\epsilon_{n}^{2}$ is minimized, while satisfying the design constraints regarding the maximum height and minimum radius of curvature. Let $h_{\max }$ be the maximum allowable height of the reflector, and $\lambda_{o}$ be the wavelength corresponding to the minimum frequency of operation. Then the constraint under which $\epsilon_{n}^{2}$ should be minimized can be written as

$$
\left(h\left(a_{e}, b_{e}, x_{m}, \gamma_{m}\right)-h_{\max }\right)^{2}=0
$$

and

$$
\left(\frac{R_{e h}\left(a_{e}, b_{e}, x_{m}, \gamma_{m}\right)-\frac{\lambda_{e}}{4}}{\lambda_{o} / 4}\right)^{2}=0
$$

where $h$ is the total height of the reflector, and $R_{s h}$ is the radius or curvature of the blended rolled edge at the incident shadow boundary ${ }^{1}$. One can use the method of Lagrange Multipliers to minimize the error $\left(\epsilon_{n}^{2}\right)$; i.e., one can optimize the following function:

$$
f=\epsilon_{n}^{2}+L_{1}\left(h-h_{\max }\right)^{2}+L_{2}\left(\frac{R_{s h}-\lambda_{o} / 4}{\lambda_{o} / 4}\right)^{2}
$$

\footnotetext{
${ }^{1}$ With a source at the focus of the reflector, one can find the incident shadow boundary on the reflector surface. One can also show that the radius of curvature of the blended rolled edge is minimum near the incident shadow boundary.
} 
where $L_{1}$ and $L_{2}$ are the Largrange multipliers. A computer program was written to optimize $f$ using the conjugate gradient method. It was found that one can fix $\gamma_{m}$ and vary $a_{e}, b_{e}$ and $x_{m}$ to minimize $f$. For all values of $\gamma_{m}$, the minimum value of $f$ was approximately the same. Thus, $\gamma_{m}$ can be fixed between $105^{\circ}$ to $180^{\circ}$ (depending upon how much the surface needs to be rolled over to allow the creeping wave to propagate around the rolled edge without diffraction) and other parameters can be found. Further, it was also observed that $f$ is not a very well behaved function in the sense that it has a lot of local minima. Thus, optimizing $f$ is not a trivial task. Then, as for any optimization problem, a study of the error $\left(\epsilon_{n}^{2}\right)$ was done. It was found that for a given reflector, while keeping $\gamma_{m}$ constant, if one computes $\epsilon_{n}^{2}$ as a function of $a_{e}$ when $b_{e}$ and $x_{m}$ are chosen such that the constraints regarding the total height (22) and minimum radius of curvature (23) are met, one obtains a curve similar to the one shown in Figure 4. Note that for large values of $a_{e}$, the error term increases very rapidly with an increase in $a_{e}$; while for small values of $a_{e}$, the increase in the error term is rather slow. Thus, if one chooses a value of $a_{e}$ which is smaller than its threshold value, as shown in Figure 4, and then chooses $b_{e}$ and $x_{m}$ to satisfy the two constraints, one will obtain a well designed rolled edge without going through the optimization process. As pointed out before, $\gamma_{m}$ can be chosen anywhere between $105^{\circ}$ and $180^{\circ}$. This approach is illustrated below for a 24 foot focal length reflector.

Let us design a blended rolled edge for a 24 foot focal length reflector. The rolled edge is to be added at a height of 15 feet $\left(\rho_{j}=15^{\prime}\right)$ and the 


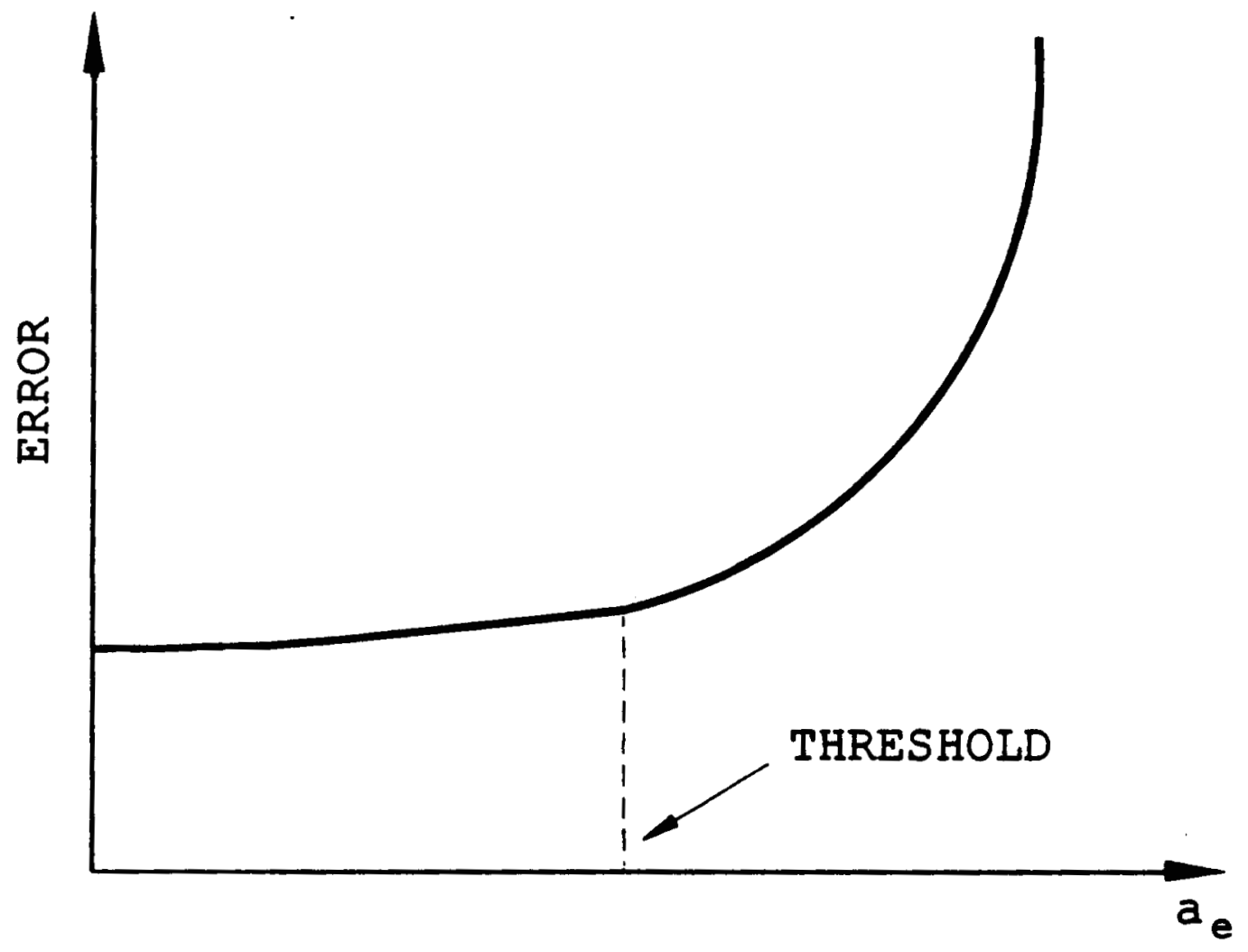

Figure 4: Error $\left(\epsilon_{n}^{2}\right)$ versus $a_{e}$ when $\gamma_{m}$ is fixed and $b_{e}$ and $x_{m}$ are chosen to satisfy the two constraints. 


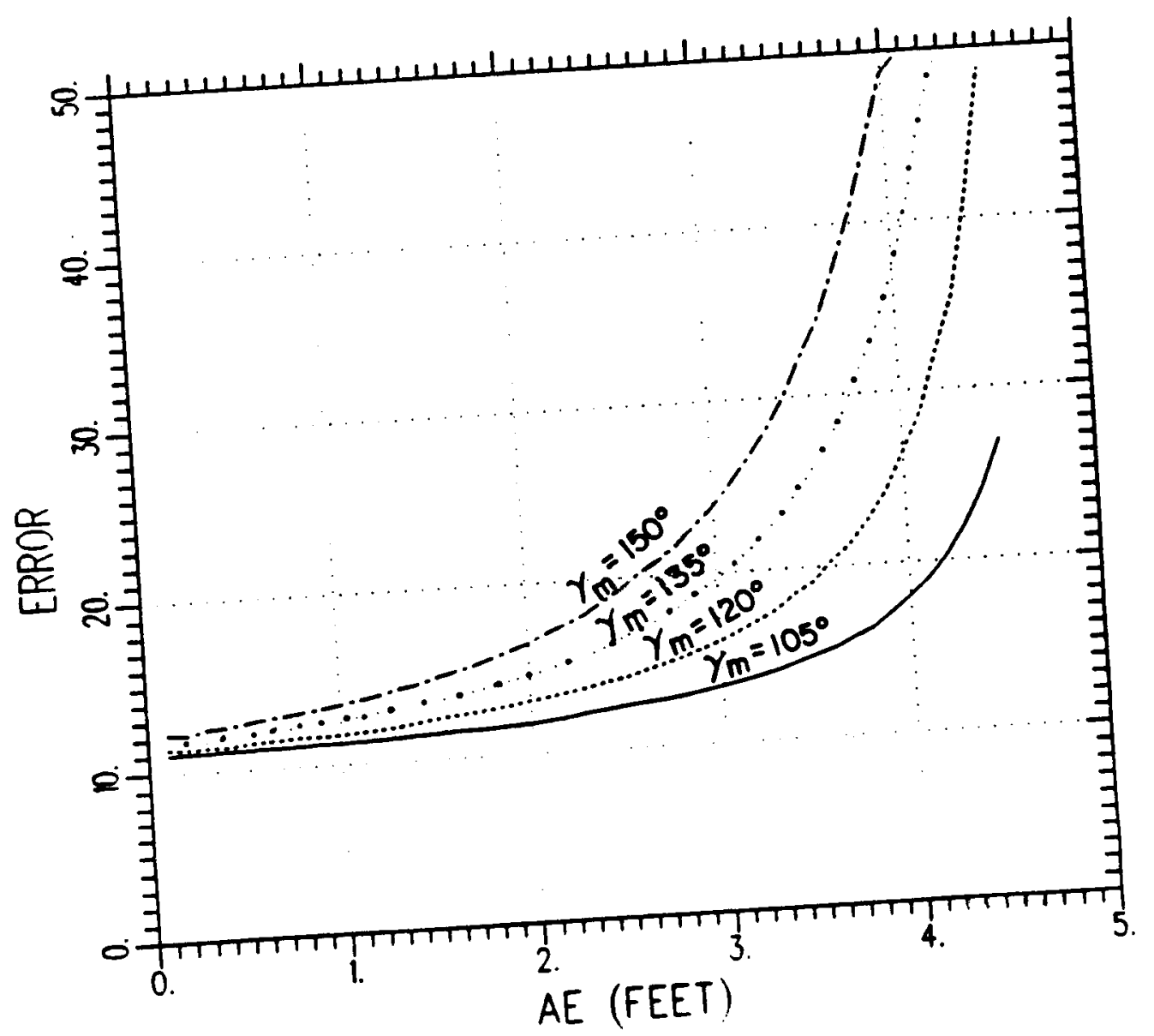

Figure 5: Error $\left(\epsilon_{n}^{2}\right)$ versus $a_{e}$ for a 24 foot focal length reflector. $\rho_{j}=15^{\prime}$, rolled edge height $=5^{\prime}$, minimum frequency $=1 \mathrm{GHz}$, cosine blending.

height of the rolled edge is limited to 5 feet. Thus, the maximum height of the reflector is 20 feet. Figure 5 shows a plot of the error term for this reflector versus $a_{e}$. The minimum frequency of operation is assumed to be $1 \mathrm{GHz}$ and the blending function is assumed to be a cosine function defined as [7]

$$
b(\gamma)=\frac{1}{2}\left(1-\cos \left(\frac{\pi \gamma}{\gamma_{m}}\right)\right)
$$

Note that the blending function is a second order function $(n=2)$ and $\alpha$ (20) for this function is equal to $12 \pi^{2}$ [7]. For the plots in Figure $5, b_{e}$ and 
Table 1: Rolled Edge Parameters for $\gamma_{m}=120^{\circ} . \quad F=24^{\prime}, \rho_{j}=15^{\prime}$, $h_{\max }=20^{\prime}$, minimum frequency $=1 \mathrm{GHz}$, cosine blending.

\begin{tabular}{|c|c|c|c|c|c|}
\hline$a_{e}$ (feet) & $b_{e}$ (feet) & $x_{m}$ (feet) & $h$ (feet) & $R_{\text {sh }}$ (feet) & $\epsilon_{n}$ \\
\hline \hline 0.1 & 4.87007 & 18.98664 & 20.00054 & 0.2458531 & 11.41249 \\
0.5 & 4.43831 & 18.59340 & 20.01333 & 0.2464865 & 11.52695 \\
1.0 & 3.888939 & 18.02378 & 20.01859 & 0.2453696 & 11.76930 \\
2.0 & 2.865024 & 16.61134 & 20.00730 & 0.2470564 & 13.12331 \\
3.0 & 1.927935 & 14.84003 & 19.99959 & 0.2458463 & 16.02044 \\
4.0 & 1.175862 & 12.35277 & 20.00961 & 0.2454030 & 26.12852 \\
4.5 & 0.9074122 & 10.38024 & 20.01350 & 0.2451355 & 47.53848 \\
\hline
\end{tabular}

$x_{m}$ for a given value of $a_{e}$ were chosen to meet the two design constraints. Plots for various values of $\gamma_{m}$ are given. Note that for small values of $a_{e}$ (below its threshold value), the error term increases very slowly with an increase in $a_{e}$ and for all values of $\gamma_{m}$ the minimum value of the error term is approximately equal. Table 1 shows the rolled edge parameters corresponding to $\gamma_{m}=120^{\circ}$ in Figure 5. Note that for all combinations of the rolled edge parameters, the total height of the reflector and $R_{s h}$ are approximately equal to the specified values.

Figure 6 shows the junction diffracted fields for some combinations of the rolled edge parameters given in Table 1. The diffracted fields are computed in front of the reflector at a $z$ distance of $\mathbf{5 0}$ feet. The reflector is assumed to be lit by a magnetic line source located at the focal point of the reflector. The frequency of operation is assumed to be $1 \mathrm{GHz}$. Corrected PO $[9,10]$ 


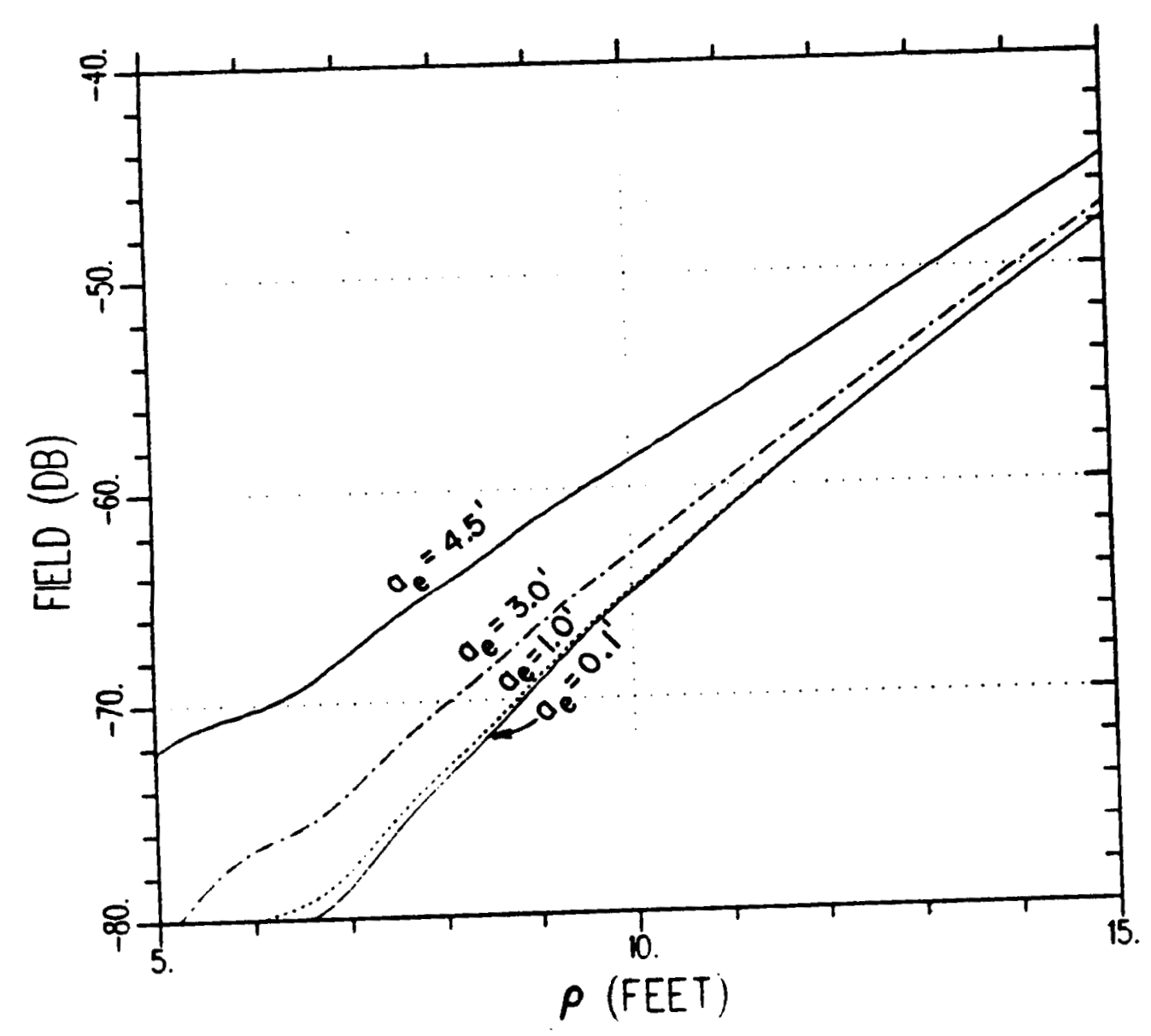

Figure 6: Junction diffracted fields for a 24 foot focal length reflector for various rolled edges. Frequency $=1 \mathrm{GHz}, z$-displacements $=50^{\prime}$ and a magnetic line source feed. 
Table 2: Rolled Edge Parameters for various junction heights. $\gamma_{m}=120^{\circ}$. $F=24^{\prime}$, minimum frequency $=1 \mathrm{GHz}$, cosine blending.

\begin{tabular}{|c|c|c|c|c|c|}
\hline$\rho_{j}$ (feet) & $a_{e}$ (feet) & $b_{e}$ (feet) & $x_{m}$ (feet) & $h$ (feet) & $\epsilon_{n}^{2}$ \\
\hline \hline-5 & 1.0 & 3.765737 & 18.27391 & 0.008 & 7.181152 \\
0. & 1.0 & 3.870553 & 17.94477 & 5.011628 & 7.786724 \\
5 & 1.0 & 3.921287 & 17.80901 & 10.01928 & 8.659282 \\
10 & 1.0 & 3.929029 & 17.82422 & 15.01887 & 10.00087 \\
15 & 1.0 & 3.888939 & 18.02378 & 20.01859 & 11.76930 \\
20 & 1.0 & 3.814528 & 18.40553 & 25.01853 & 14.03492 \\
25 & 1.0 & 3.709114 & 18.97642 & 30.02068 & 16.75188 \\
\hline
\end{tabular}

was used to compute the total scattered fields of the reflector. To obtain the junction diffracted fields, the specularly reflected fields (GO term) were subtracted from the total scattered fields. Note that the magnitude of the junction diffracted fields decreases with a decrease in $a_{e}$. However, for small values of $a_{e}$ (below threshold), the improvement is very marginal. Thus, if the rolled edge parameters are chosen corresponding to the values of $a_{e}$ which are smaller than its threshold value, one obtains a good set of rolled parameters and can avoid the optimization process.

An important point that should be mentioned here is that the optimum rolled edge parameters depend on the junction height. For example, Table 2 shows the rolled edge parameters when the junction height $\left(\rho_{j}\right)$ is varied. All other parameters are the same as before. The height of the rolled edge is still limited to 5 feet. Note that $b_{e}$ and $x_{m}$ varies with the junction height 
and so does the error term $\left(\epsilon_{n}^{2}\right)$. Thus, if the junction height is changed one should obtain a new set of rolled edge parameters. Blended rolled edges for 3-dimensional reflectors are discussed next.

\section{BLENDED ROLLED EDGES FOR 3-D REFLECTORS}

Let a paraboloid of focal length $F$ be defined in the (xyz) coordinate system as shown in Figure 7. Then the defining equation for the paraboloid is given by

$$
z=\frac{x^{2}+y^{2}}{4 F}
$$

A section of this paraboloid is used as the main reflector for a compact range application. The reflector can have any rim shape. Let us call this rim the 'junction contour'. If one wishes to add a blended rolled edge to this reflector, he should make sure that the total surface of the reflector is smooth and continuous. Therefore, the choice of the rolled edge plane for various points on the junction contour is very important. Such a plane is described below. If the rolled edge is added in this plane one obtains a unique (single valued) surface which is smooth and continous. This rolled edge plane is applicable for center-fed as well as offset-fed compact range reflectors.

Let $\left(x_{\text {avg }}, y_{\text {avg }}, z_{\text {avg }}\right)$ be the center of the main reflector. Note that for a center-fed system, the center of the reflector coincides with the origin of 


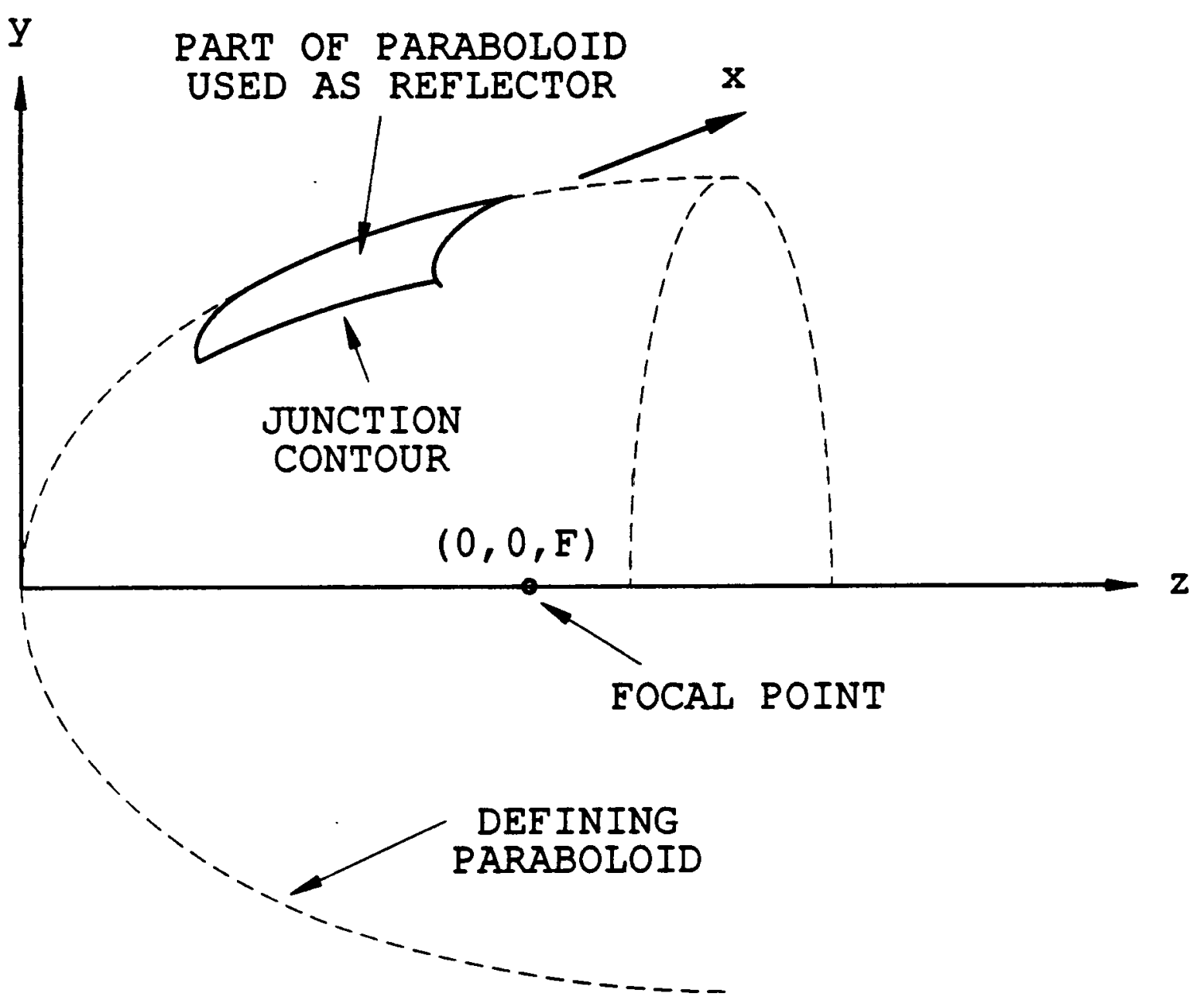

Figure 7: Paraboloid coordinate system and the junction contour for a compact range reflector. 
the $(x y z)$ coordinate system. Further,

$$
z_{a v g}=\left(x_{a v g}^{2}+y_{a v g}^{2}\right) / 4 F
$$

Let us define a new coordinate system $\left(x^{\prime} y^{\prime} z^{\prime}\right)$ whose origin is the center of the main reflector. Note that the new coordinate system is obtained by translating the original coordinate system, and the transformation between the two coordinate systems is given by

$$
\left.\begin{array}{l}
x=x^{\prime}+x_{\text {avg }} \\
y=y^{\prime}+y_{\text {avg }}, \text { and } \\
z=z^{\prime}+z_{\text {avg }}
\end{array}\right\} \text {. }
$$

Next a cylindrical coordinate system $\left(\rho^{\prime} \phi^{\prime} z^{\prime}\right)$ with its origin coinciding with the origin of the $\left(x^{\prime} y^{\prime} z^{\prime}\right)$ coordinate system can be defined, as shown in Figure 8. Note that the transformation between the $\left(x^{\prime} y^{\prime} z^{\prime}\right)$ and the $\left(\rho^{\prime} \phi^{\prime} z^{\prime}\right)$ coordinate system is given by

$$
\left.\begin{array}{l}
x^{\prime}=\rho^{\prime} \cos \phi^{\prime} \\
y^{\prime}=\rho^{\prime} \sin \phi^{\prime} \\
z^{\prime}=z^{\prime}
\end{array}\right\} .
$$

Let $\rho_{j}^{\prime}\left(\phi^{\prime}\right)$ be the junction contour of the reflector in $\left(\rho^{\prime} \phi^{\prime} z^{\prime}\right)$ coordinate system. Then the defining equation of the parabolic part of the main reflector is

$$
\begin{array}{r}
\rho^{\prime 2}+2 \rho^{\prime}\left(x_{\text {avg }} \cos \phi^{\prime}+y_{\text {avg }} \sin \phi^{\prime}\right)=4 F z^{\prime} \\
\text { for }\left\{\begin{array}{l}
0 \leq \rho^{\prime} \leq \rho_{j}^{\prime}\left(\phi^{\prime}\right) \\
0 \leq \phi^{\prime} \leq 2 \pi
\end{array}\right\} .
\end{array}
$$

Note that for most of the reflector surfaces $\rho_{j}^{\prime}\left(\phi^{\prime}\right)$ is a single valued function of $\phi^{\prime}$. Then if for a given point on the rim ( $\phi^{\prime}$ is fixed), the rolled edge is 


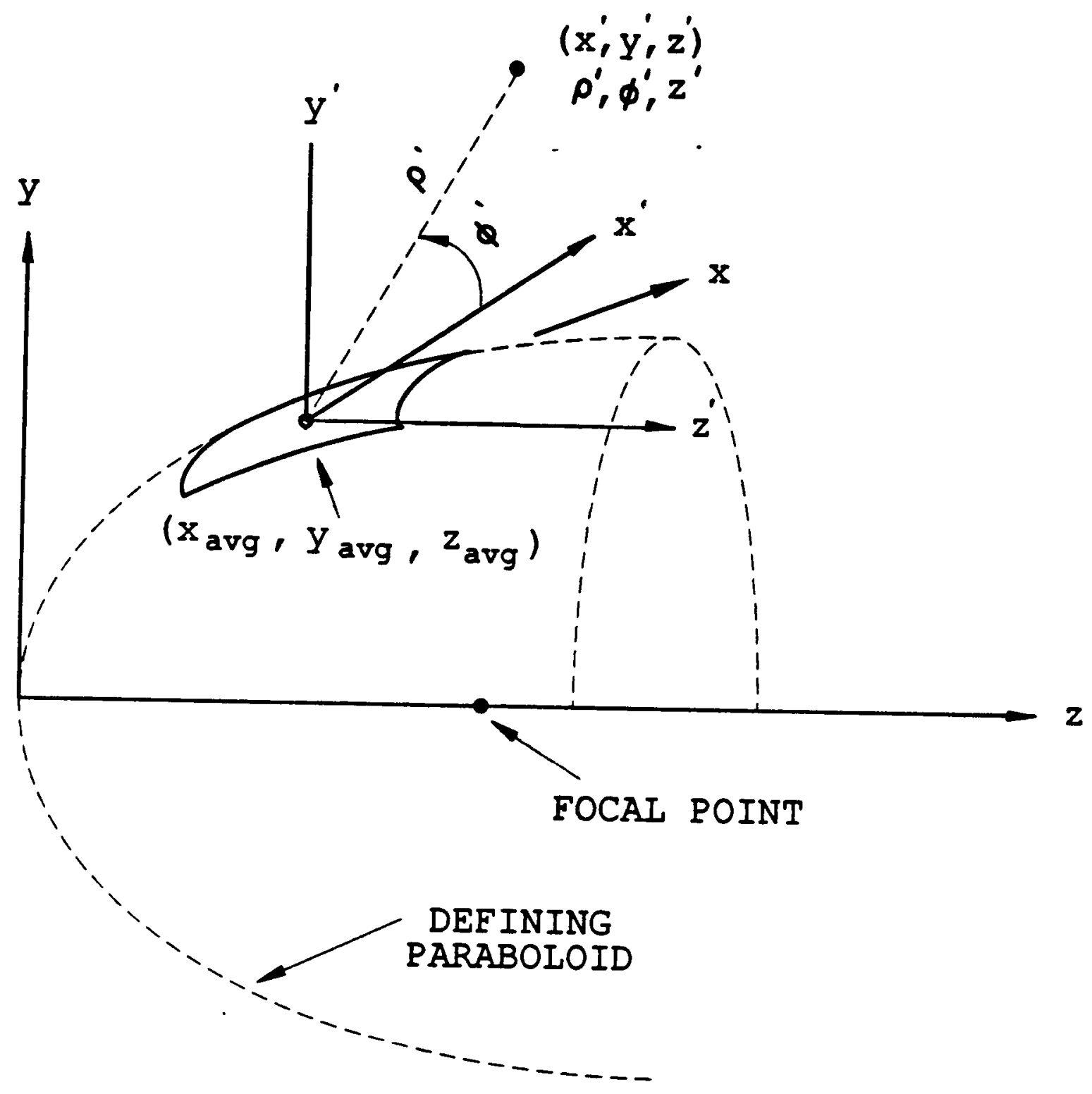

Figure 8: Various coordinate systems. $\left(x_{a v g}, y_{\text {avg }}, z_{\text {avg }}\right)$ is the center of the compact range reflector. 
added in $\rho^{\prime} z^{\prime}$ plane, the total surface will be smooth and continuous. Also if the junction contour is a single valued function of $\phi^{\prime}$, which is normally true, the rolled edge surface will be unique. Once the rolled edge plane is defined, one can use the method outlined in Section II to add the rolled edge; while, the method outlined in Section III can be used to obtain the rolled edge parameters. However, to use the method given in Sections II and III, part of the paraboloid which lies in the $\rho^{\prime} z^{\prime}$ plane (the rolled edge plane) should be a parabola and one should know the focal length of the parabola and the junction height. As shown below, the part of the paraboloid in the rolled edge plane is a parabola, and it is trivial to find junction height and the focal length of the parabola. Let us assume that the rolled edge is added at a point corresponding to $\phi^{\prime}=\phi_{j}^{\prime}$. Then the equation of the part of the paraboloid in the rolled edge plane becomes

$$
\rho^{\prime 2}+2 \rho^{\prime}\left(x_{a v g} \cos \phi_{j}^{\prime}+y_{a v g} \sin \phi_{j}^{\prime}\right)=4 F z^{\prime}
$$

Note that Equation (31) represents a parabola of focal length $(F)$. The vertex of the parabola is at

$$
\left[-\left(x_{a v g} \cos \phi_{j}^{\prime}+y_{a v g} \sin \phi_{j}^{\prime}\right),-\frac{\left(x_{a v g} \cos \phi_{j}^{\prime}+y_{a v g} \sin \phi_{j}^{\prime}\right)^{2}}{4 F}\right] .
$$

Thus, the junction height in the rolled edge plane, as shown in Figure 9, is

$$
\rho_{j}^{\prime \prime}=\rho_{j}^{\prime}\left(\phi_{j}^{\prime}\right)+x_{a v g} \cos \phi_{j}^{\prime}+y_{a v g} \sin \phi_{j}^{\prime}
$$

Now one can use the procedure outlined in the last two sections to add the rolled edge and obtain the rolled edge parameters. Note that since the 


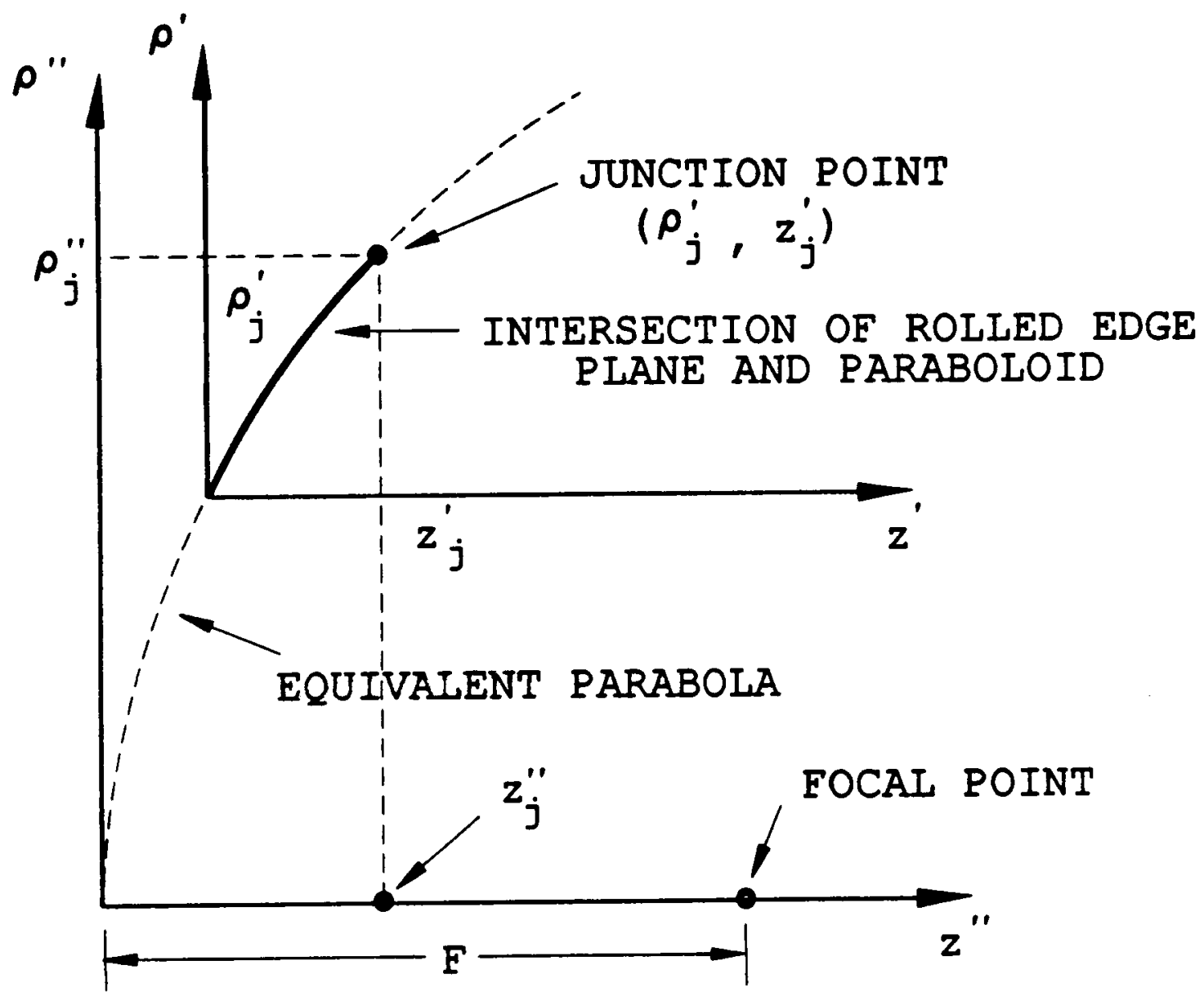

Figure 9: The section of the paraboloid in the rolled edge plane $\left(\rho^{\prime} z^{\prime}\right)$. Vertex of the equivalent parabola coincides with the origin of $\left(\rho^{\prime \prime} z^{\prime \prime}\right)$ coordinate system. 
height of the junction, $\rho_{j}^{\prime \prime}$, may vary with $\phi_{j}^{\prime}$, the rolled edge parameters will vary with $\phi_{j}^{\prime}$. From Section II, the rolled edge in the $\left(\rho^{\prime} z^{\prime}\right)$ coordinate system is defined by

$$
\begin{array}{r}
\rho^{\prime}(\gamma)=\left[\gamma \frac{x_{m}\left(\phi_{j}^{\prime}\right)}{\gamma_{m}\left(\phi_{j}^{\prime}\right)} x_{p 2}\right][1-b(\gamma)]+\left[\left(a_{e}\left(\phi_{j}^{\prime}\right) \sin \gamma\right) x_{p 2}\right. \\
\left.+b_{e}\left(\phi_{j}^{\prime}\right)(1-\cos \gamma) y_{p 2}\right] b(\gamma)+\rho_{j}^{\prime}\left(\phi_{j}^{\prime}\right)
\end{array}
$$

and

$$
\begin{aligned}
z^{\prime}(\gamma)= & {\left[\left(\gamma \frac{x_{m}\left(\phi_{j}^{\prime}\right)}{\gamma_{m}\left(\phi_{j}^{\prime}\right)} x_{p 2}+\rho_{j}^{\prime \prime}\right)^{2} / 4 F\right][1-b(\gamma)] } \\
& +\left[\left(a_{e}\left(\phi_{j}^{\prime}\right) \sin \gamma\right) x_{p 3}+b_{e}\left(\phi_{j}^{\prime}\right)(1-\cos \gamma) y_{p 3}+z_{j}^{\prime \prime}\right] b(\gamma)- \\
& \left(x_{a v g} \cos \phi_{j}^{\prime}+y_{a v g} \sin \phi_{j}^{\prime}\right)^{2} / 4 F
\end{aligned}
$$

where

$$
\begin{gathered}
z_{j}^{\prime \prime}=\left(\rho_{j}^{\prime \prime}\right)^{2} / 4 F \\
x_{p 2}=2 F /\left(\rho_{j}^{\prime{ }^{2}}+4 F^{2}\right)^{1 / 2} \\
y_{p 2}=\rho_{j}^{\prime \prime} /\left(\rho_{j}^{\prime \prime 2}+4 F^{2}\right)^{1 / 2} \\
x_{p 3}=\rho_{j}^{\prime \prime} /\left(\rho^{\prime \prime^{2}}+4 F^{2}\right)^{1 / 2}, \quad \text { and } \\
y_{p 3}=-2 F /\left(\rho_{j}^{\prime \prime^{2}}+4 F^{2}\right)^{1 / 2} .
\end{gathered}
$$

Thus, the whole surface is defined analytically in the $\left(\rho^{\prime} \phi^{\prime} z^{\prime}\right)$ coordinate system. For given $\phi^{\prime}$, Equation (30) defines the surface for $\rho^{\prime} \leq \rho_{j}^{\prime}\left(\phi^{\prime}\right)$; while 
for $\rho>\rho_{j}^{\prime}\left(\phi^{\prime}\right)$, Equations (34) and (35) define the surface as a function of parametric angle, $\gamma$.

Next, a blended rolled edge for a concave rim reflector $[7,8]$ (see Appendix) is designed. The focal length of the reflector is 7.25 feet, and the reflector is offset in the $y$ direction by 8.5 feet; i.e., $x_{\text {avg }}=0$ and $y_{a v g}=8.5^{\prime}$. The scattered fields of the reflector in the target area are also computed.

\section{DESIGN EXAMPLE}

Figure 10 shows the front view of the junction contour of the reflector. Note that the junction contour is concave in shape. Equations defining a concave rim reflector are given in the Appendix. The reflector is symmetric about the $y$ axis and the target zone extends from $-4^{\prime}$ to $4^{\prime}$ in the $x$-direction and from $5.5^{\prime}$ to $11.5^{\prime}$ in the $y$-direction. Figure 11 shows the height of the junction in the rolled edge plane $\left(\rho^{\prime} z^{\prime}\right)$ versus $\phi^{\prime}$. Note that the junction height varies with $\phi^{\prime}$ and goes from a large positive value to a large negative value. However, the variation is quite smooth and reasonable. Thus, one can optimize the rolled edge parameters for a few points around the rim and then use interpolation for the rest. Figure 12 shows the total reflector surface obtained using this process. The rolled edge height is limited to 3.5 feet and the concavity parameters, $r_{e}$ (see Appendix), is also chosen to be 3.5 feet. The minimum frequency of operation is assumed to be $2 \mathrm{GHz}$. A cosine squared blending function [7] is used to blend the elliptical rolled 


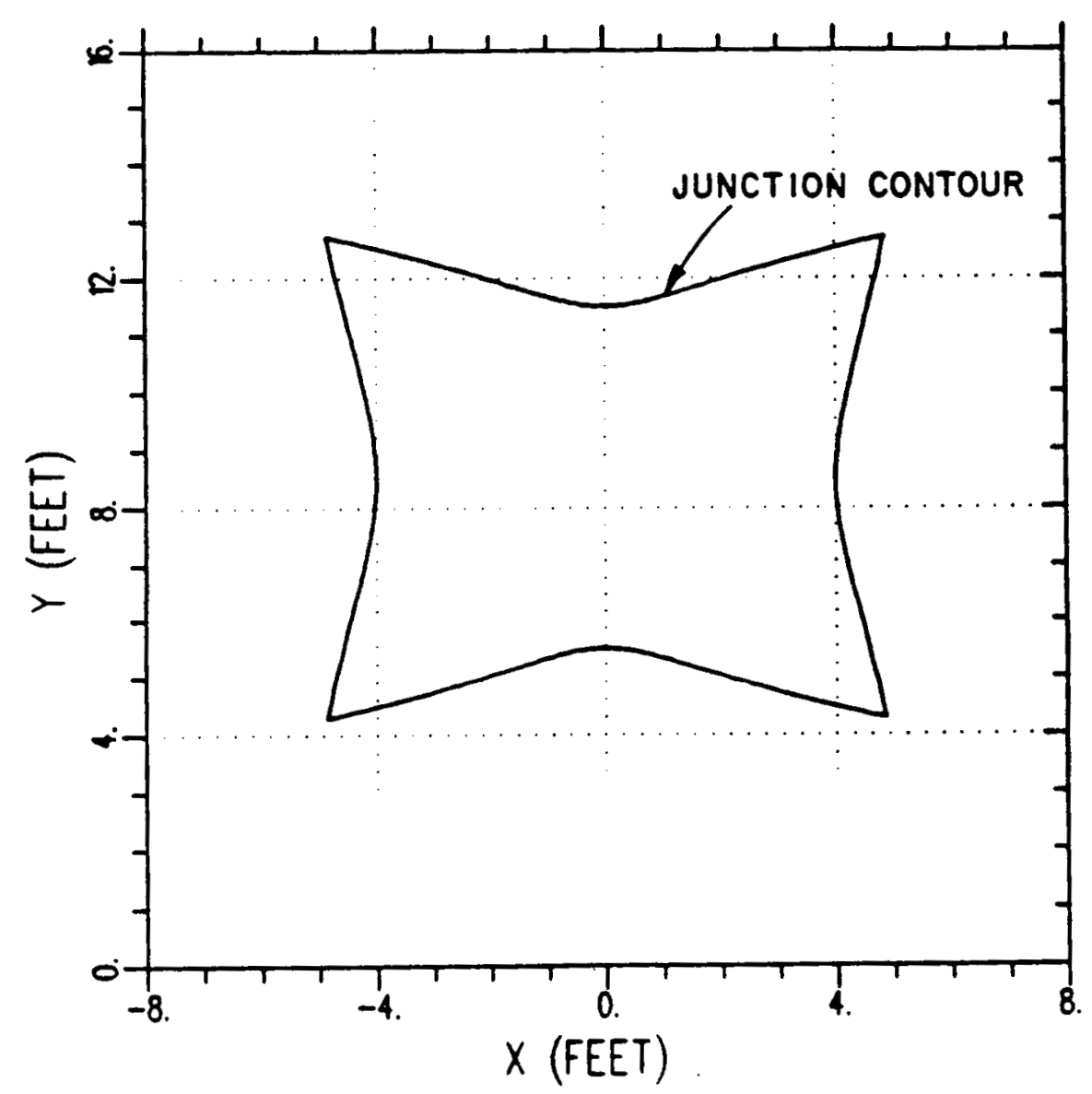

Figure 10: Front view of the junction contour of a concave edge compact range reflector with $x_{\text {left }}=-4^{\prime}, x_{\text {right }}=4^{\prime}, y_{\text {bottom }}=5.5^{\prime}$, and $y_{\text {top }}=11.5^{\prime}$. The focal length is $24^{\prime}$ and $r_{e}=3.5^{\prime}$. 


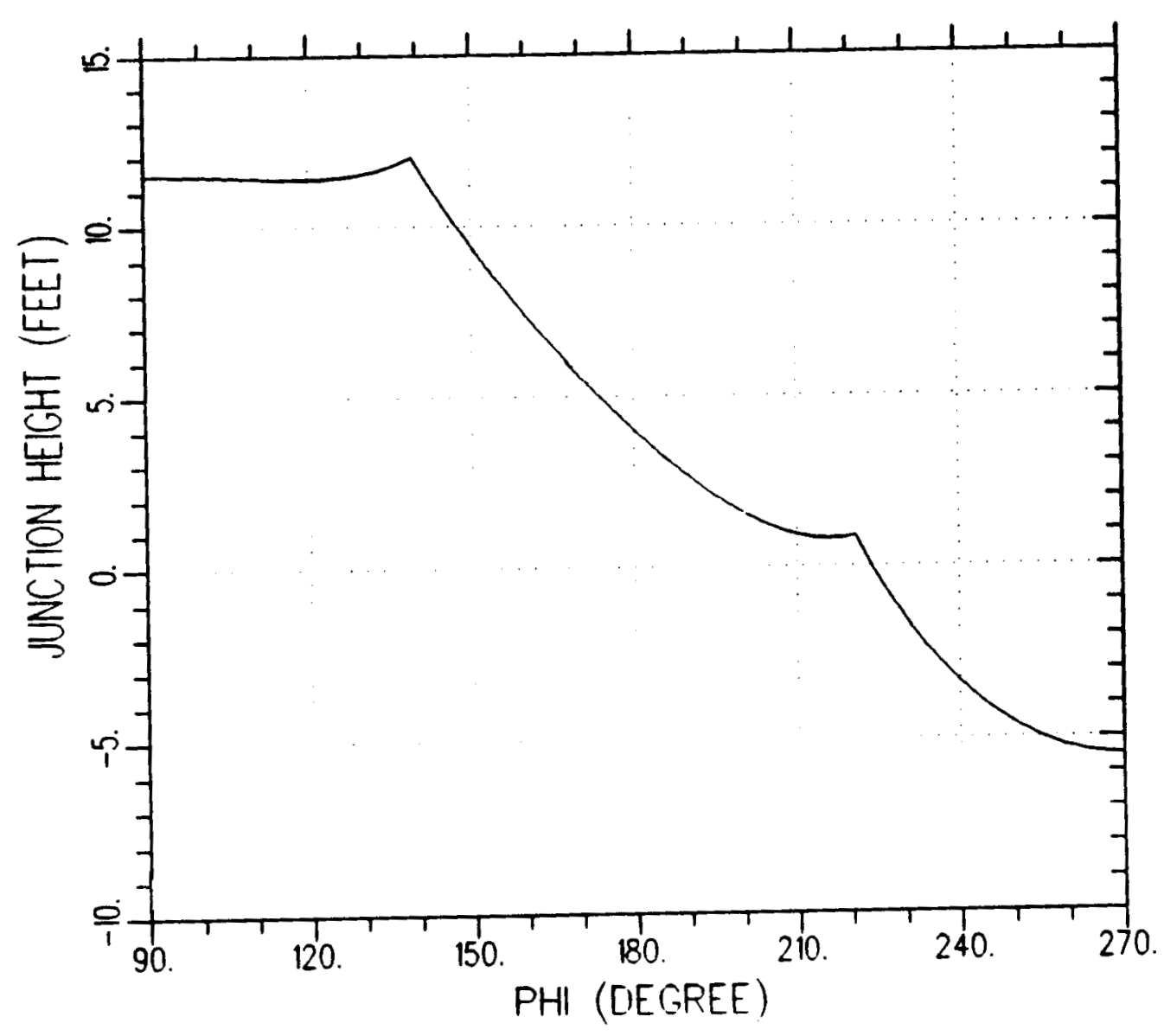

Figure 11: Junction height versus $\phi^{\prime}$ for the concave edge reflector. 


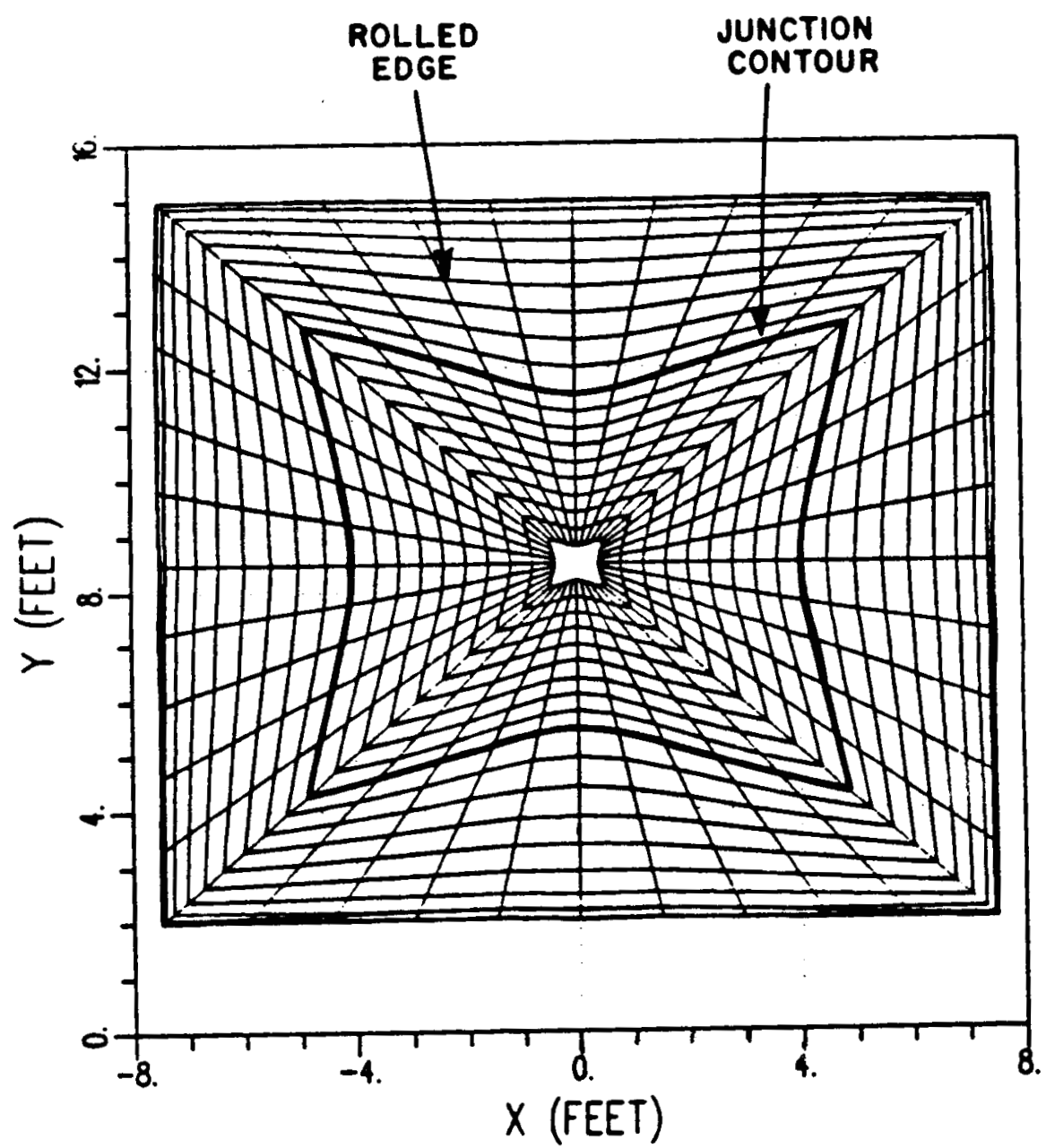

Figure 12: Front view of the concave edge reflector with cosine squared blended rolled edges. Rolled edge height $=3.5^{\prime}$, minimum frequency $=2$ $\mathrm{GHz}$. 
Table 3: Rolled Edge Parameters for the Concave Edge Reflector. $F=7.25$ feet, minimum frequency $=2 \mathrm{GHz}$, cosine squared blending.

\begin{tabular}{|c|c|c|c|c|}
\hline$\phi^{\prime}$ (deg.) & $a_{e}$ (feet) & $b_{e}$ (feet) & $x_{m}$ (feet) & $\gamma_{m}$ (deg.) \\
\hline \hline $90^{\circ}$ & 0.5 & 2.7984 & 10.8642 & $105^{\circ}$ \\
$118^{\circ}$ & 0.5 & 2.8128 & 10.8162 & $105^{\circ}$ \\
$139.0856^{\circ}$ & 0.5 & 2.7396 & 10.9962 & $105^{\circ}$ \\
$160^{\circ}$ & 0.5 & 3.2809 & 9.6723 & $105^{\circ}$ \\
$180^{\circ}$ & 0.5 & 3.6719 & 9.0867 & $105^{\circ}$ \\
$200^{\circ}$ & 0.5 & 3.9026 & 8.9810 & $105^{\circ}$ \\
$216^{\circ}$ & 0.5 & 3.9620 & 9.0146 & $105^{\circ}$ \\
$220.9144^{\circ}$ & 0.5 & 3.9574 & 9.0111 & $105^{\circ}$ \\
$226.0^{\circ}$ & 0.5 & 4.0061 & 9.0927 & $105^{\circ}$ \\
$234.0^{\circ}$ & 0.5 & 3.9897 & 9.3187 & $105^{\circ}$ \\
$250.0^{\circ}$ & 0.5 & 3.8700 & 9.8004 & $105^{\circ}$ \\
$270.0^{\circ}$ & 0.5 & 3.7929 & 10.0663 & $105^{\circ}$ \\
\hline
\end{tabular}

edge and the paraboloid. The function is defined as

$$
b(\gamma)=\frac{1}{4}\left(1-\cos \frac{\pi \gamma}{\gamma_{m}}\right)^{2}
$$

It can be shown that the function is a fourth order blending function. Note that the total reflector surface is smooth and continuous. The whole reflector fits in a $15^{\prime} \times 13^{\prime}$ rectangle and is symmetrical about the $y$-axis. Table 3 shows the rolled edge parameters for a few points along the junction contour. Recall that for other points the rolled edge parameters were obtained by interpolation. Since the reflector is symmetric about the $y$-axis, the rolled edge parameters are only given for $90^{\circ} \leq \phi^{\prime} \leq 270^{\circ}$. 


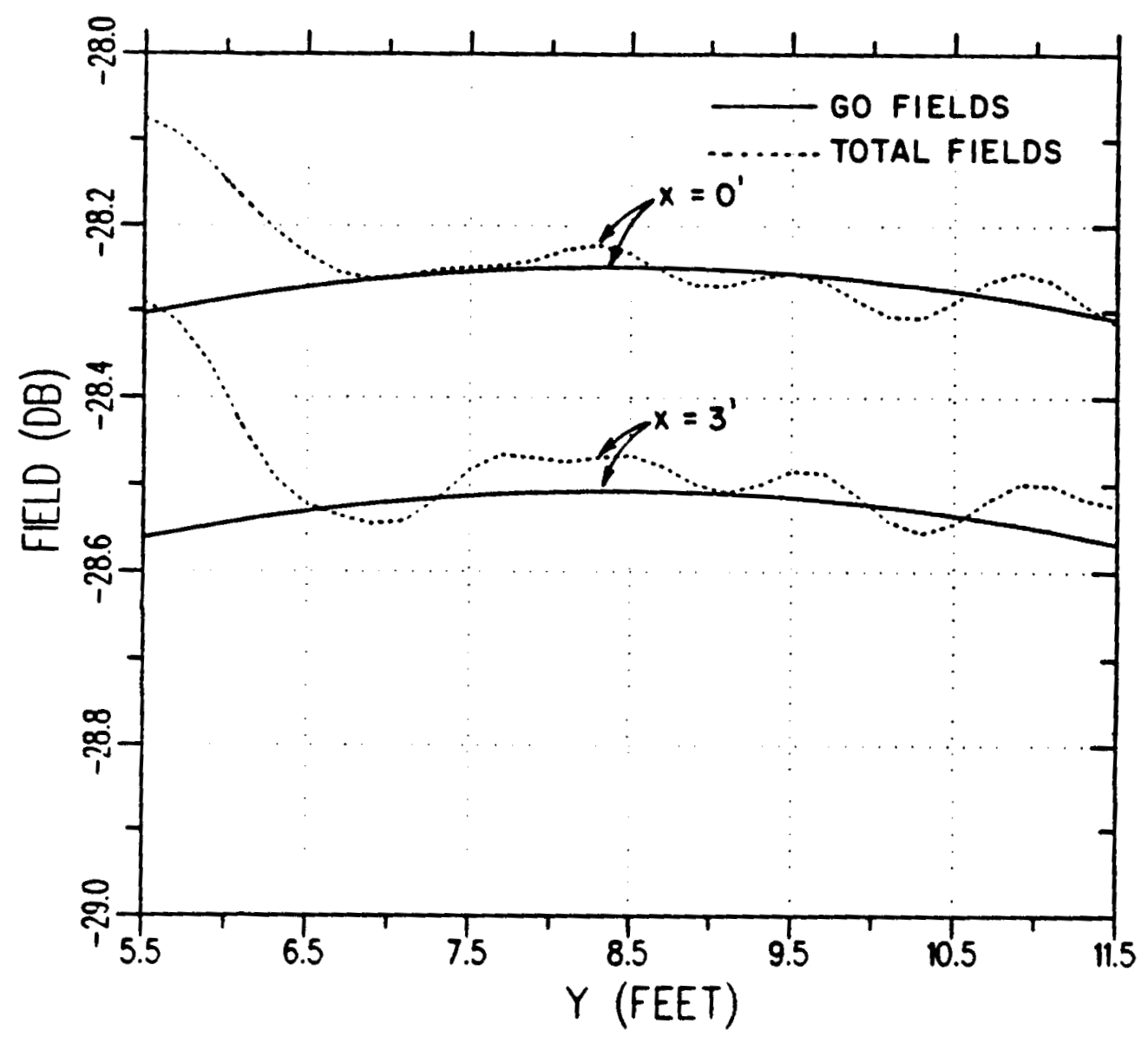

Figure 13: Scattered fields of the concave edge reflector in the target zone along two vertical cuts. $z$-displacement $=20^{\prime}$, Frequency $=2 \mathrm{GHz}$, Subreflector feed.

Figure 13 shows the scattered fields for this reflector along two vertical cuts. The frequency of operation is $2 \mathrm{GHz}$, and the $z$-displacement for the fields cuts is $20^{\prime}$ from the vertex of the paraboloid. The $x$-displacements for the two vertical cuts is $0^{\prime}$ and $3^{\prime}$, respectively. The reflector is assumed to be illuminated by an elliptical subreflector (Gregorian System). The subreflector axis and the feed were tilted such that the cross-polarization for a Huygen's source feed is zero [11]. The subreflector parameters are 


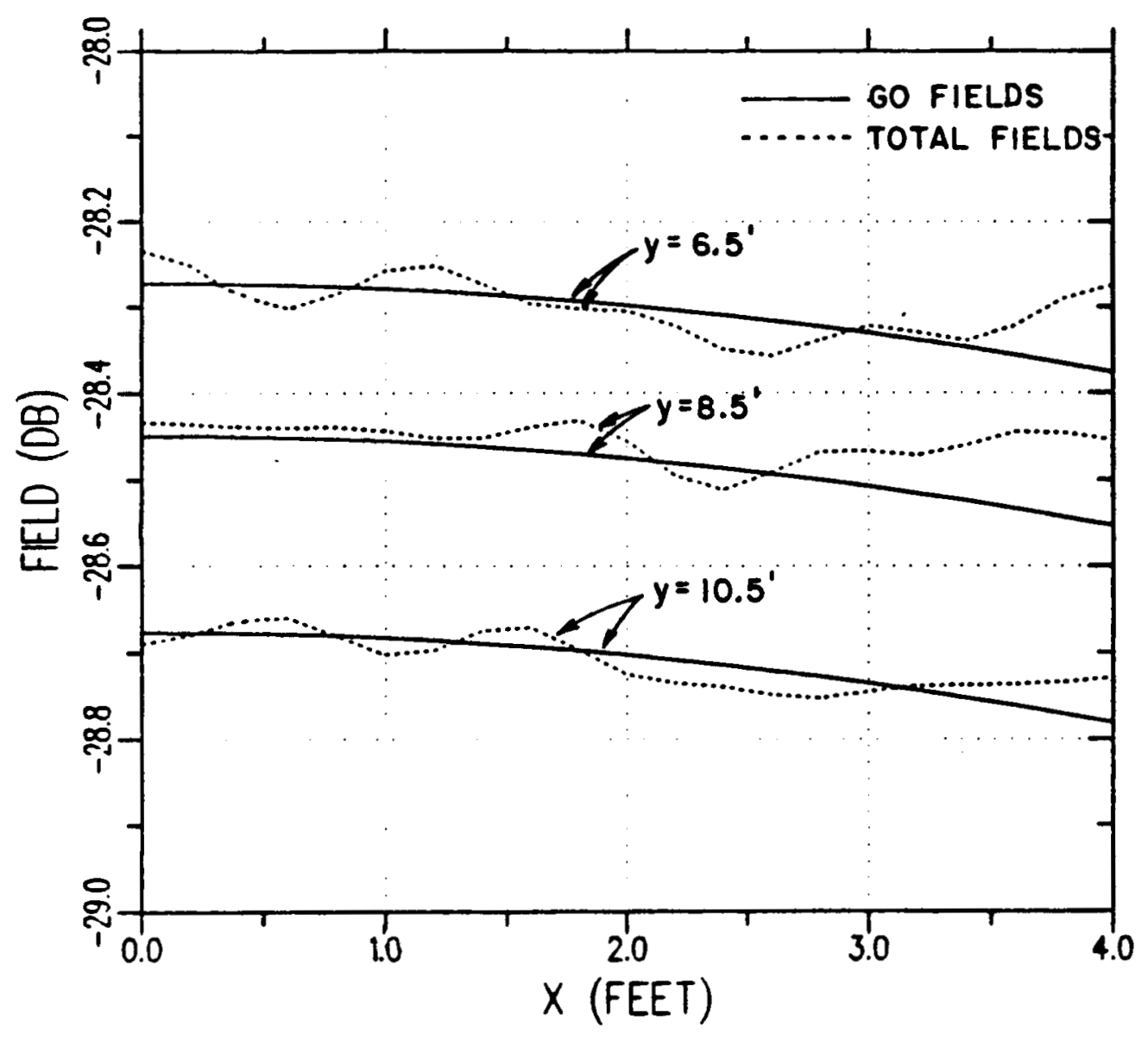

Figure 14: Scattered fields of the concave edge reflector along three horizontal cuts. $z$-displacement $=20^{\prime}$, Frequency $=2 \mathrm{GHz}$, Subreflector feed.

$a_{s}=5.25^{\prime}, b_{s}=4.308^{\prime}, \beta=5.5^{\circ}$ and $\alpha=20^{\circ}[12]$. Corrected PO [10] is used to compute the scattered fields. For $x=3^{\prime}$, the fields are displaced by $-0.2 \mathrm{~dB}$ so that the two results can be seen in isolation of each other. For comparison, the GO field level is also shown in the figure. Note that in most of the target zone, the ripple in the scattered fields is less than 0.1 $\mathrm{dB}$, which is excellent for even the most stringent applications.

Figure 14 shows the scattered fields along three horizontal cuts $\left(y=6.5^{\prime}\right.$, $8.5^{\prime}$ and $10.5^{\prime}$ ). All other parameters are the same as before. For the $8.5^{\prime}$ and $10.5^{\prime}$ field cuts, the scattered fields are displaced by $-0.2 \mathrm{~dB}$ and -0.4 
$\mathrm{dB}$, respectively. Since the reflector system is symmetrical about the $y$ axis, the scattered fields only for positive values of $x$ are computed. For comparison, GO field levels are also plotted in the figure. Again, in most of the target zone; the ripple in the scattered fields is less than $0.1 \mathrm{~dB}$, which is very good. Thus, the design procedure leads to blended rolled edges which cause very small junction diffracted fields in the target zone.

\section{SUMMARY AND CONCLUSION}

A method to obtain optimum rolled edge parameters for elliptical blended rolled edges was given. The method ensures that the total height of the reflector does not exceed the specified value and the minimum radius of curvature of the reflector meets the requirement at the lowest frequency of operation. The method also guarantees small diffracted fields.

A procedure to add blended rolled edges to arbitrary rim shaped 3dimensional compact range reflectors was also given. The procedure is applicable to center-fed as well as offset-fed reflectors and leads to rolled edges with minimal surface discontinuities. Using the procedure, the whole reflector surfact can be defined analytically using simple expressions. It was demonstrated that the design procedure leads to reflector surfaces which have very small diffracted fields emanating from the junction between the paraboloid and the rolled edge surface. 


\section{APPENDIX}

Let the desired target zone extend from $x_{l e f t}$ to $x_{\text {right }}$ in the $x$-direction and from $y_{\text {bottom }}$ to $y_{\text {top }}$ in the $y$-direction. Then a concave edge is formed by the locii of points that are a radial distance $r_{e}$ away from the border of a rectangle (defining rectangle) which extends from $x_{l e f t}-r_{e}$ to $x_{r i g h t}+r_{e}$ in the $x$-direction and from $y_{\text {bottom }}-r_{e}$ to $y_{\text {top }}+r_{e}$ in the $y$-direction $[7,8]$, as shown in Figure 15. The coordinates of the points on the concave edge contour are given by

$$
\begin{gathered}
x_{j}=\left\{\begin{array}{cc}
x_{\text {right }}+r_{e}\left(1-\cos \phi^{\prime}\right) & 0 \leq \phi^{\prime} \leq \phi_{1} \\
x_{\text {avg }}+\left(y_{\text {top }}+r_{e}-y_{\text {avg }}\right) \cot \phi^{\prime}-r_{e} \cos \phi^{\prime} & \phi_{1} \leq \phi^{\prime} \leq \phi_{2} \\
x_{\text {left }}-r_{e}\left(1+\cos \phi^{\prime}\right) & \phi_{2} \leq \phi^{\prime} \leq \phi_{3} \\
x_{\text {avg }}+\left(y_{\text {bottom }}-r_{e}-y_{\text {avg }}\right) \cot \phi^{\prime}-r_{e} \cos \phi^{\prime} & \phi_{3} \leq \phi^{\prime} \leq \phi_{4} \\
x_{\text {right }}+r_{e}\left(1-\cos \phi^{\prime}\right) & \phi_{4} \leq \phi^{\prime} \leq 360^{\circ}
\end{array}\right. \\
y_{j}=\left\{\begin{array}{cc}
y_{a v g}+\left(x_{\text {right }}+r_{e}-x_{a v g}\right) \tan \phi^{\prime}-r_{e} \sin \phi^{\prime} & 0 \leq \phi^{\prime} \leq \phi_{1} \\
y_{\text {top }}+r_{e}\left(1-\cos \phi^{\prime}\right) & \phi_{1} \leq \phi^{\prime} \leq \phi_{2} \\
y_{a v g}+\left(x_{\text {left }}-r_{e}-x_{a v g}\right) \tan \phi^{\prime}-r_{e} \sin \phi^{\prime} & \phi_{2} \leq \phi^{\prime} \leq \phi_{3} \\
y_{b o t t o m}-r_{e}\left(1+\cos \phi^{\prime}\right) & \phi_{3} \leq \phi^{\prime} \leq \phi_{4} \\
y_{a v g}+\left(x_{\text {right }}+r_{e}-x_{a v g}\right) \tan \phi^{\prime}-r_{e} \sin \phi^{\prime} & \phi_{4} \leq \phi^{\prime} \leq 360^{\circ}
\end{array}\right. \\
z_{j}=\left(x_{j}^{2}+y_{j}^{2}\right) / 4 F
\end{gathered}
$$

where

$$
\begin{array}{r}
x_{\text {avg }}=\left(x_{\text {left }}+x_{\text {right }}\right) / 2 \\
y_{\text {avg }}=\left(y_{\text {top }}+y_{\text {bottom }}\right) / 2 \\
\phi_{1}=\tan ^{-1}\left(\frac{y_{\text {top }}+r_{e}-y_{\text {avg }}}{x_{\text {right }}+r_{e}-x_{\text {avg }}}\right) \\
\phi_{2}=\tan ^{-1}\left(\frac{y_{\text {top }}+r_{e}-y_{\text {avg }}}{x_{\text {left }}-r_{e}-x_{\text {avg }}}\right)
\end{array}
$$



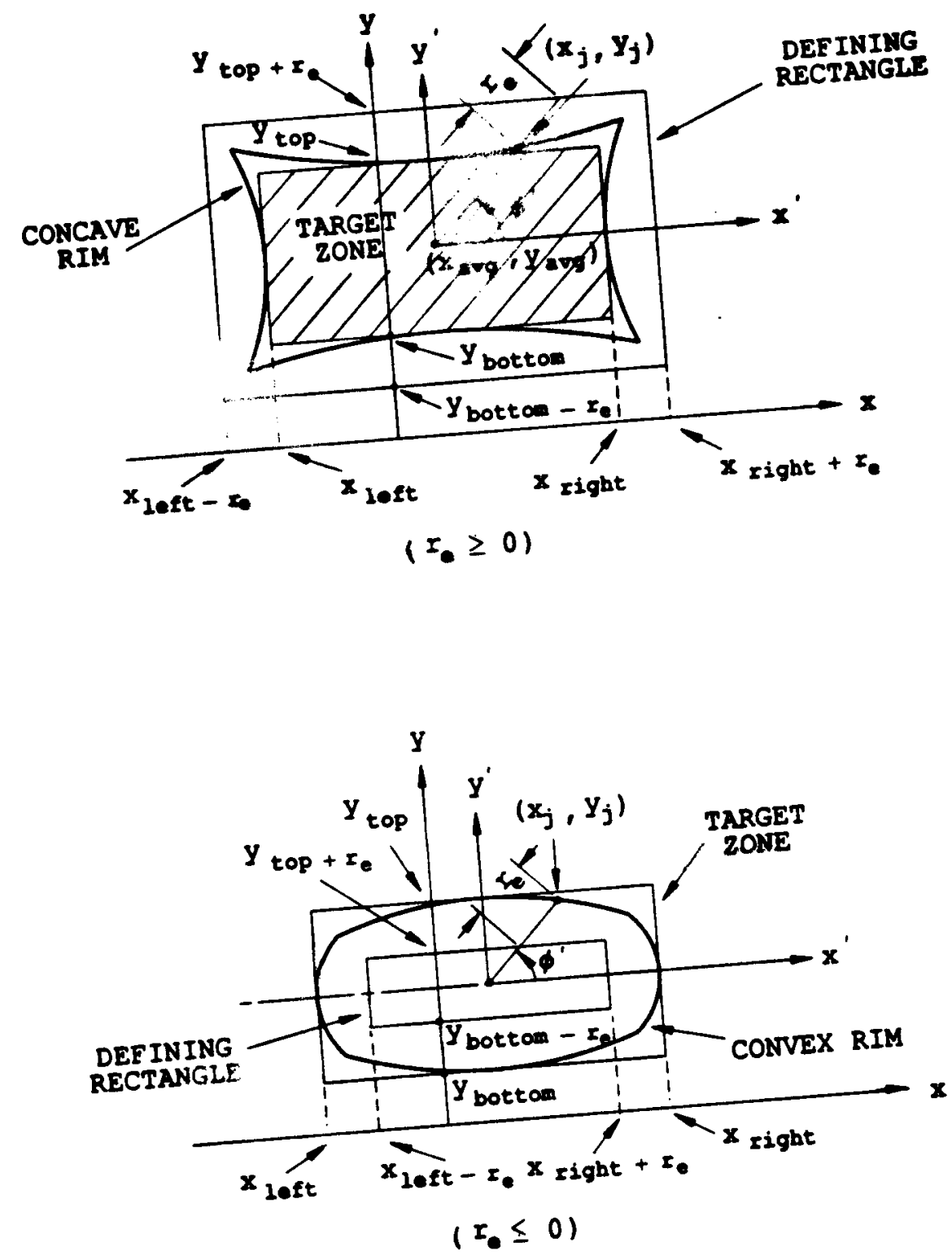

Figure 15: Target zone, defining rectangle and the junction contour. 


$$
\begin{gathered}
\phi_{3}=\tan ^{-1}\left(\frac{y_{\text {bottom }}-r_{e}-y_{\text {avg }}}{x_{\text {left }}-r_{e}-x_{\text {avg }}}\right) \\
\phi_{4}=\tan ^{-1}\left(\frac{y_{\text {bottom }}-r_{e}-y_{\text {avg }}}{x_{\text {right }}+r_{e}-x_{\text {avg }}}\right) .
\end{gathered}
$$

For a concave rim $r_{e}$ should be positive. Note that the parameter $r_{e}$ controls the concavity. The concavity increases with an increase in $r_{e}$. It can be shown that if $r_{e}$ is chosen to be equal to the height of the rolled edge, the $x y$ projection of the main reflector source (including rolled edge), will extend from $x_{l e f t}-r_{e}$ to $x_{r i g h t}+r_{e}$ in the $x$ direction and from $y_{b o t t o m}-r_{e}$ to $y_{t o p}+r_{e}$ in the $y$ direction. If $r_{e}=0$, one gets a rectangular rim. For $r_{e}<0$, one obtains a convex rim (see Figure 15). 


\section{References}

[1] R.C. Johnson, H.A. Ecker, and R.A. Moore, "Compact Range Techniques and Measurements," IEEE Trans. Antennas and Propagation, Vol. AP-17, No. 5, pp. 568-576, May 1969.

[2] R.C. Johnson and D.W. Hess, "Performance of a Compact Antenna Range," Proceeding of IEEE Antenna and Propagation Society, International Symposium, 1975.

[3] E.B. Joy and R.E. Wilson, "Shaped Edge Serrations for Improved Compact Range Performance," Proceeding of Antenna Measurement Techniques Association's 1987 Meeting, Seattle, Washington, September 28 - October 2, 1987.

[4] V. Galindo, "Design of Dual-Reflector Antennas with Arbitrary Phase and Amplitude Distributions," IEEE Trans. Antennas and Propagation, Vol. AP-12, No. 7, pp. 403-408, July 1964.

[5] W.D. Burnside, M.C. Gilreath, B.M. Kent and G.L. Clerici, "Curved Edge Modification of Compact Range Reflectors," IEEE Transactions on Antennas and Propagation, Vol. AP-35, No. 2, pp. 176-182, February 1987.

[6] W.D. Burnside, A.K. Dominek and R. Barger, "Blended Surface Concept for a Compact Range Reflector," Proceeding of the Antenna Measurement Techniques Association's 1985 meeting, Melbourne, Florida, September 1985 .

[7] C.W.I. Pistorius, New Main Reflector, Subreflector and Dual Chamber Concept for Compact Range Applications, Ph.D. dissertation, The Ohio State University, Dept. of Electrical Engineering, Columbus, Ohio, 1986. 
[8] C.W.I. Pistorius and W.D. Burnside, "An Improved Main Reflector Design for Compact Range Applications," IEEE Transactions on Antennas and Propagation, Vol. AP-35, No. 3, pp. 342-347, March 1987.

[9] I.J. Gupta and W.D. Burnside, "A Physical Optics Correction for Backscattering from Curved Surfaces," IEEE Trans. Antennas and Propagation, Vol. AP-35, No. 5, pp. 553-561, May 1987.

[10] I.J. Gupta, C.W.I. Pistorius and W.D. Burnside, "An Efficient Method to Compute Spurious End Point Contributions in PO Solutions," IEEE Transactions on Antennas and Propagation, Vol. AP-35, No. 12, pp. 1426-1435, December 1987.

[11] R.A. Shore, "A Simple Derivation of the Basic Design Equation for Offset Dual Reflector Antennas with Rotational Symmetry and Zero Cross Polarization," IEEE Transactions on Antennas and Propagation, Vol. AP-33, No. 1, pp. 114-116, January 1985.

[12] C.W.I. Pistorius, G. Clerici and W.D. Burnside, "A Dual chamber Gregorian Subreflector System for Compact Range Applications," accepted for publication in IEEE Transactions on Antennas and Propagation. 